\title{
Handover in Mobile WiMAX Networks: The State of Art and Research Issues
}

\author{
Sayan Kumar Ray, Krzysztof Pawlikowski, Senior Member, IEEE, and Harsha Sirisena, Senior Member, IEEE
}

\begin{abstract}
The next-generation Wireless Metropolitan Area Networks, using the Worldwide Interoperability for Microwave Access (WiMAX) as the core technology based on the IEEE 802.16 family of standards, is evolving as a Fourth-Generation (4G) technology. With the recent introduction of mobility management frameworks in the IEEE 802.16e standard, WiMAX is now placed in competition to the existing and forthcoming generations of wireless technologies for providing ubiquitous computing solutions. However, the success of a good mobility framework largely depends on the capability of performing fast and seamless handovers irrespective of the deployed architectural scenario. Now that the IEEE has defined the Mobile WiMAX (IEEE 802.16e) MAC-layer handover management framework, the Network Working Group (NWG) of the WiMAX Forum is working on the development of the upper layers. However, the path to commercialization of a full-fledged WiMAX mobility framework is full of research challenges. This article focuses on potential handover-related research issues in the existing and future WiMAX mobility framework. A survey of these issues in the MAC, Network and Cross-Layer scenarios is presented along with discussion of the different solutions to those challenges. A comparative study of the proposed solutions, coupled with some insights to the relevant issues, is also included.
\end{abstract}

Index Terms-Mobile WiMAX, IEEE 802.16e, Handover, MAC-layer, IP-layer, Cross-layer, Issues.

\section{INTRODUCTION}

$\mathbf{T}$ HE steady global boom in the number of users of the global Internet has led to the development of different fixed and mobile broadband technologies providing support for high speed streaming multimedia, customized personalized services, ubiquitous coverage and unhampered QoS. Though the existing Wireless Local Area Network (WLAN) and third generation $(3 \mathrm{G})$ technologies have been successfully providing broadband access for the last several years, they have their specific drawbacks, prohibiting their full-fledged growth. WLANs suffer from short range and restricted scalability. On the other hand, the $3 \mathrm{G}$ systems have such constraints as low bandwidth and high infrastructural expenses. The culmination of the recent IEEE 802.16-based WiMAX family of standards (IEEE 802.16a, 16d and 16e) for Wireless Metropolitan Area Networks has filled this gap between the LAN and WAN technologies. Devised as a truly broadband access solution, the WiMAX technology offers promising features in terms of high bandwidth, extended coverage area and low cost. This

Sayan K. Ray and K. Pawlikowski are with the Department of Computer Science and Software Engineering, University of Canterbury, Christchurch, New Zealand, e-mail: (skr29@student.canterbury.ac.nz, krys.pawlikowski@canterbury.ac.nz).

H. Sirisena is with the Department of Electrical and Computer Engineering, University of Canterbury, Christchurch, New Zealand, e-mail: (harsha.sirisena@canterbury.ac.nz). has led to its fast rise as one of the most popular last mile broadband access technologies and as a likely component in the $4 \mathrm{G}$ networks. While the OFDM-based IEEE 802.16d [1] technology (commonly termed fixed WiMAX) provides fixed broadband access from anywhere within a metropolitan area network, the new mobile air interfaces specified in the IEEE 802.16e [2] (commonly termed mobile WiMAX) has successfully addressed the requirements for higher data rates and efficient spectral efficiencies in provisioning full-fledged mobile broadband access. An IEEE 802.16e-based Base Station (BS) can support both fixed and mobile broadband wireless access.

Similar to the different cellular and broadband technologies, global mobility related research in WiMAX is mostly focused on two main areas of concern: location management and handover management. In the former, the underlying network technology tracks and maintains the exact whereabouts of wireless terminals in cases when they are powered-on, powered-off or even on the move. On the other hand, the latter deals with the active transfer of wireless terminals from the control of a BS in one cell to the control of another BS in a different cell. Handovers can be broadly classified into two different types depending on the underlying technology: horizontal handovers and vertical handovers. Horizontal handovers are homogeneous intra-network inter-cellular, while the vertical ones are heterogeneous inter-network inter-cellular. For example, handovers between multiple WiMAX networks are horizontal, whereas those between WiMAX and 3G or WLAN networks are vertical. In this paper, we focus on the homogeneous handover management. Mobility aspects in WiMAX are specified as an individual Mobility Agent (MA) layer, above the MAC (link) layer, with some network layer signaling to develop a complete solution. The existing WiMAX mobility structure defines three types of link layer handover procedures in a homogeneous environment. Of these, Hard Handover (HHO) is the default handover mechanism and two soft handover mechanisms, Macro-Diversity Handover (MDHO) and Fast Base Station Switching (FBSS), are the optional procedures. The standard specifies a highly flexible and scalable layer 2 (MAC-layer) handover policy, allowing handovers to be initiated and optimized by the mobile station (MS), the BS or the backbone network. Facilities are there to support all types of probable handover activities like intra- and inter-cell, intra- and inter-sector, inter-layer, as well as intraand inter-system.

The existing WiMAX handover mechanisms suffer from certain drawbacks, particularly related to wastage of channel resources, handover latencies and loss of data. According to [3]-[4], WiMAX is envisioned to support low-latency seamless 
handovers of much less than $100 \mathrm{~ms}$ and almost zero packet loss, with an MS speed of $120 \mathrm{~km} / \mathrm{h}$ or more during the handover activity. The global telecommunication sector is quite positive that WiMAX technology has the potential to achieve this performance. However, several mobility and handover related research issues must be resolved before the potential of WiMAX is realized. Every step in the technological advancement of WiMAX from the standardization of its network layer mobility architecture to devising an universally accepted crosslayer handover management (CLHM) framework, presents considerable challenges. The already standardized MAC-layer mobility and handover framework may also raise certain research issues. Furthermore, in addition to internal challenges, WiMAX also faces competition from technologies such as 3GPP Long Term Evolution (LTE) [5]. The different handover related WiMAX research issues need to be resolved, both to allow WiMAX to fulfil its potential and to ensure that it sees more widespread adoption.

The aim of this paper, to the best of our knowledge the first of its kind, is to give an overview of these potential issues along with the different proposed and probable research solutions, starting right from the advent of IEEE 802.16e technology until today, thus identifying the research directions related to the existing and future WiMAX homogeneous handover scenarios. In this article, we will focus on the mobile WiMAX technology and will use the acronym 'MWiMAX' instead of mobile WiMAX in the rest of the paper. A list of the different acronyms used in the paper is provided in Appendix A.

The rest of the article is organized as follows. In section II, we briefly recapitulate the different handover techniques in MWiMAX and present a comparative study of the advantages of the different handover procedures. This is followed in section III by a brief discussion about the different potential deployment architectures of the MWiMAX technology and their relevancy with the write-up. Section IV presents an overview to the comparative study between MWiMAX and LTE technologies. The MWiMAX link layer, network layer and cross-layer (layer 2+3) homogeneous handover issues, with insights to the proposed and possible solutions to each of them, are then categorized and discussed in detail in section V. The article finishes with Conclusion in section VI.

\section{MWIMAX HANDOVER SCENARIO}

The IEEE 802.16 standardization group has defined three types of approaches towards handover for the 802.16e technology [2] depicted in Figure 1. While HHO is the default handover procedure, FBSS and MDHO are the optional types. In MWiMAX, a handover initiation decision by a wireless terminal or BS is dependent on the Received Signal Strengths (RSS) from the current serving BS (SBS) and the neighbouring BSs (NBS). The MS and the SBS jointly decide on when to initiate a handover activity. Whenever the RSS from the SBS drops below a certain threshold, which might hamper an ongoing communication session, the MS goes for a handover with one of the chosen NBSs, called the target BS (TBS).

The HHO [Figure 1(a)], is a Break-Before-Make (BBM) procedure, in which the MS breaks its communication with the SBS before getting connected with the TBS. Thus, the MS experiences a communication gap between its termination from the previously connected $\mathrm{BS}$ and the reconnection to the new targeted BS. On the other hand, both MDHO [Figure 1(b)] and FBSS [Figure 1(c)] are of the Make-Before-Break (MBB) type (soft handover), where the MS starts communicating with the new BS before terminating its service with the previous BS. Clearly, these latter two types of handover procedure do not experience any gaps in the ongoing communication and the MS remains connected to multiple BSs simultaneously. Although the different handover techniques in IEEE 802.16e have been designed from the layer 2 handover perspective, both FBSS and MDHO, which are seamless and fast in nature, can provide support for even higher-layer handovers. The next sub-sections briefly describe the three handover procedures.

\section{A. Hard Handover}

The entire process of HHO in IEEE 802.16e is broadly divided into Network Topology Acquisition Phase (NTAP) and the Actual Handover phase (AHOP). Detailed explanation of the entire procedure can be found in [2].

Network Topology Acquisition Phase: During the NTAP, the MS and serving BS (SBS), together with the help of the backhaul network, gather information about the underlying network topology before the actual handover decision is made. This is done to identify lists of potential NBSs, out of which one particular TBS may be chosen for the handover activity. Figure 2 shows the message sequence chart for the procedure. The major tasks involved in this phase are briefly as follows:

- BS advertises the Network Topology: Using MOB_NBRADV (Mobile Neighbour Advertisement) message, the SBS periodically broadcasts information about the state of the NBSs, preparing for potential handover activities. The SBS keeps on gathering these channel information of the NBSs with the help of the backbone network.

- Scanning of advertised neighbouring BSs by MS: The MS scans the advertised BSs within specific time frames, to select suitable candidate BSs for the handover. A list of potential candidate TBSs is thus maintained. This procedure is carried out with the help of Scanning Interval Allocation request and response messages (MOB_SCN-REQ and MOB_SCN-RSP), respectively, sent by the MS and the SBS. In the end, Scanning Result Report (MOB_SCN-REP) summarizes all the scanning activities.

- Ranging and Optional Association Activities: The scanning is followed by contention/non-contention ranging activities through which the MS gathers further information about the PHY channel related to the selected TBSs. Ranging Request (RNG_REQ) and Ranging Response (RNG_RSP) messages are used for this purpose. Ranging may be followed by optional 


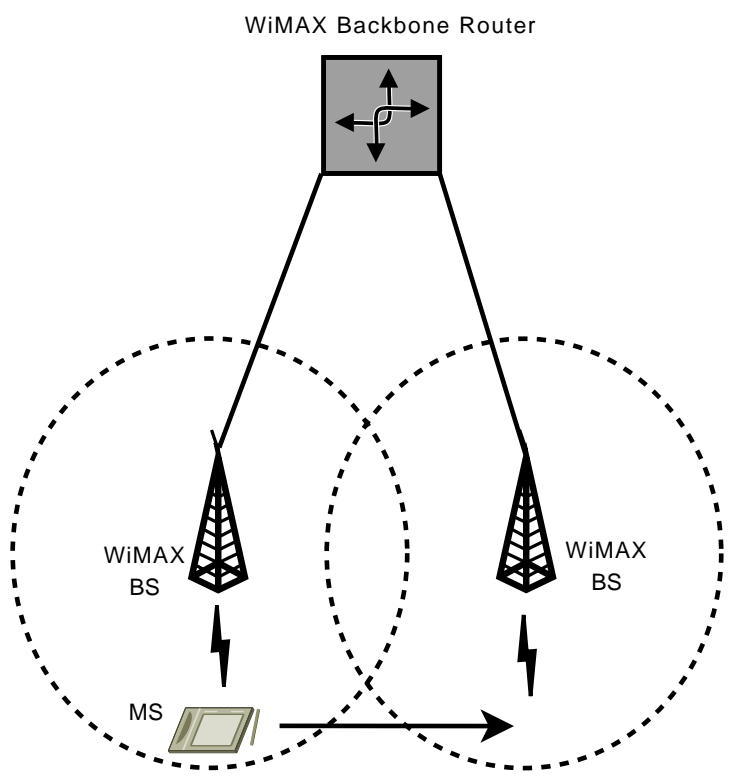

(a) $\mathrm{HHO}$

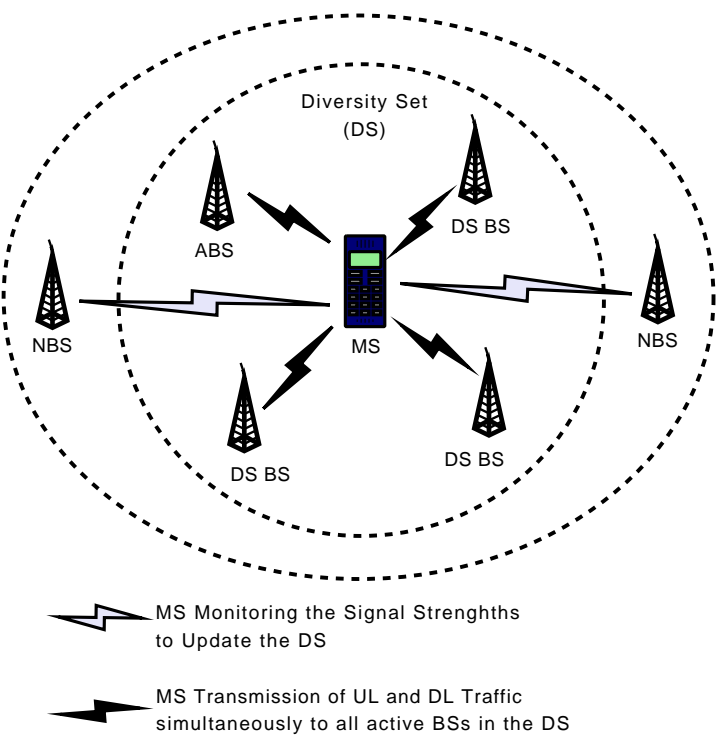

(b) $\mathrm{MDHO}$

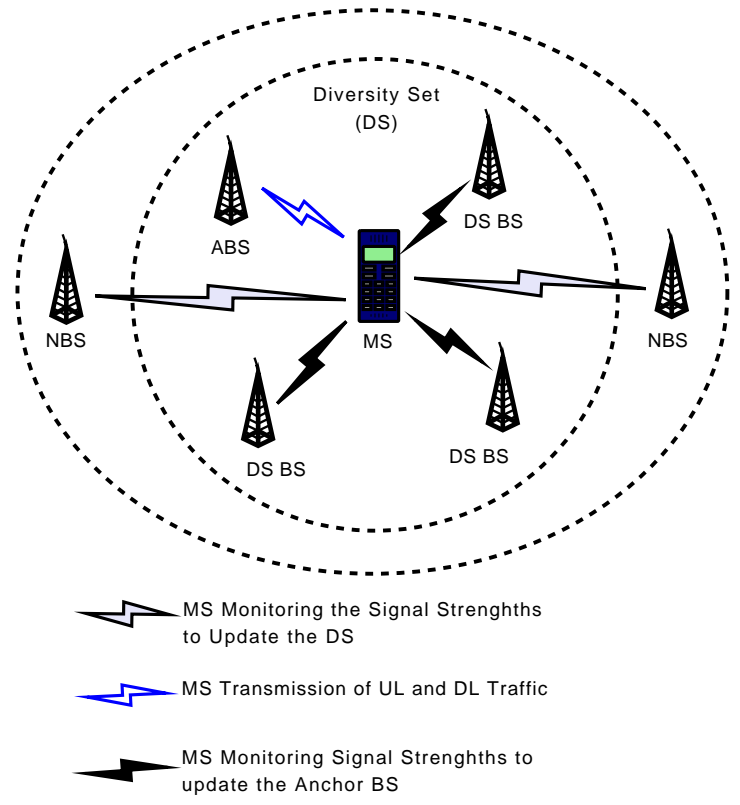

(c) FBSS

Fig. 1. MWiMAX Handover Procedures (a) HHO (b) MDHO [6] (c) FBSS [6]

association activities through which the MS gets associated with the potential target BS candidates. Association Result Reports (MOB_ASC-REP) are used for this purpose.

Actual Handover Phase: During the AHOP (Figure 3), the MS switches location from the SBS to the selected TBS. The major tasks involved are briefly described as follows:

- Deciding on the TBS: Here the MS chooses the final TBS to handover to, out of the multiple TBSs selected from the scanning activities. The decision or initialization of a handover process may arise at the MS, the SBS or at the network associated. If the decision arises at the
MS, it communicates the MOB_MSHO-REQ message containing the list of selected TBSs to the SBS and the SBS replies back with the MOB_BSHO-RSP message. On the other hand, if the decision arises at the SBS, the MOB_BSHO-REQ message is used. However, handover decision and initiation messages from the MS are always given preference.

- Initiating the Handover: Depending on the abovementioned messages, once a particular TBS is selected from the list of the suitable candidate TBSs, the MS informs the current SBS about the beginning of the HO activity by sending a MOB_HO-IND (Mobile Handover Indication) message. It is at this point that the 


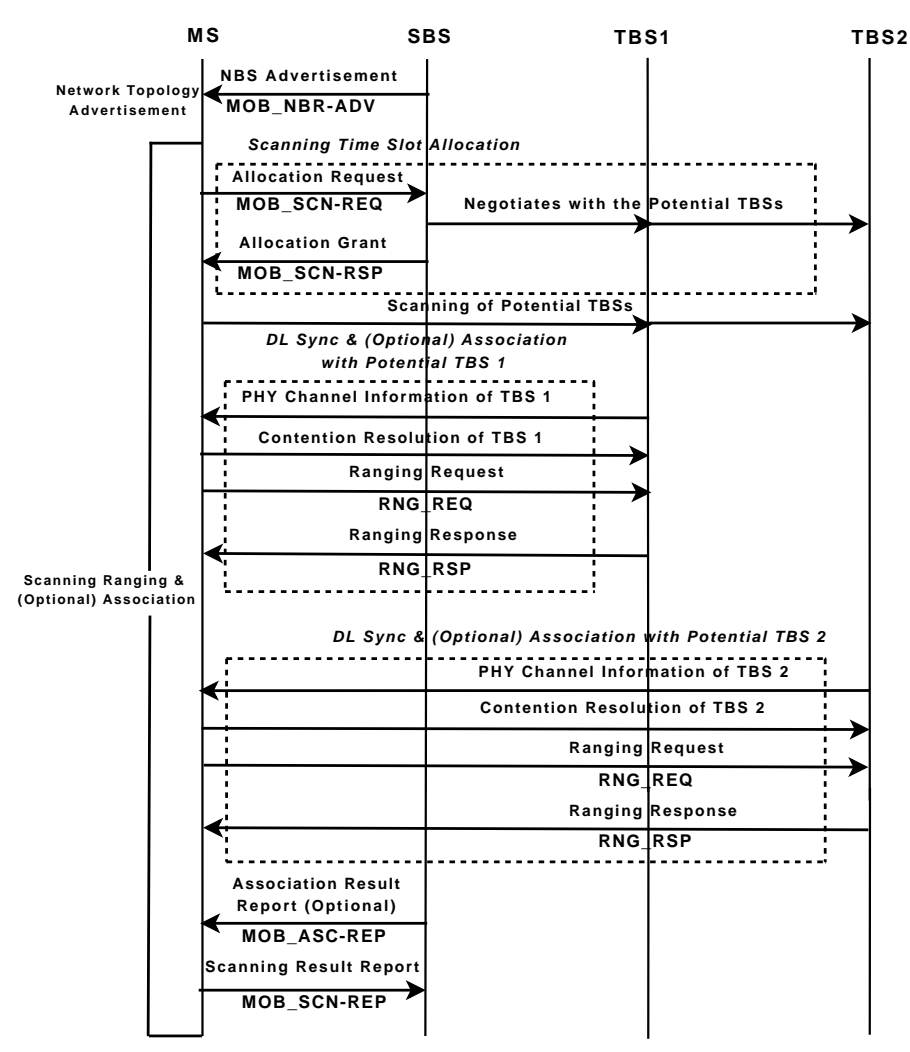

Fig. 2. NTAP Message Sequence Charts

MS terminates its connection with the current SBS.

- TBS synchronization and Ranging Process: Appropriate synchronization and ranging activities take place once again with the TBS, to resume DL/UL retransmissions.

- Authorization and Registration Phases: Lengthy authorization and registration processes of the MS with the TBS follow next. It marks the onset of the network reentry phase of this MS, after which it becomes fully functional with the new SBS.

\section{B. Macro Diversity Handover and Fast Base Station Switching}

In the case of the optional handover approaches, MDHO and FBSS, the MS simultaneously communicates using the air interfaces of multiple BSs, i.e. the MS is connected to multiple BSs at a time, unlike the HHO procedure in which the MS remains connected to single BS at any instant. Both the MDHO and the FBSS use the concepts of Diversity Set (DS) and Anchor BS (ABS). Each MS has a DS.

At any time, depending on the signal strengths, the DS includes the most active NBSs that could be involved in a handover. The ABS is chosen as the one with the most powerful signal strength (the most active BS). In case of the MDHO, each MS simultaneously communicates with all the BSs in its DS. However, in FBSS, the MS communicates only with the ABS during the downlink (DL) and uplink (UL) activities. So, signal strengths of neighbouring BSs are continuously monitored by each MS for efficient updating of

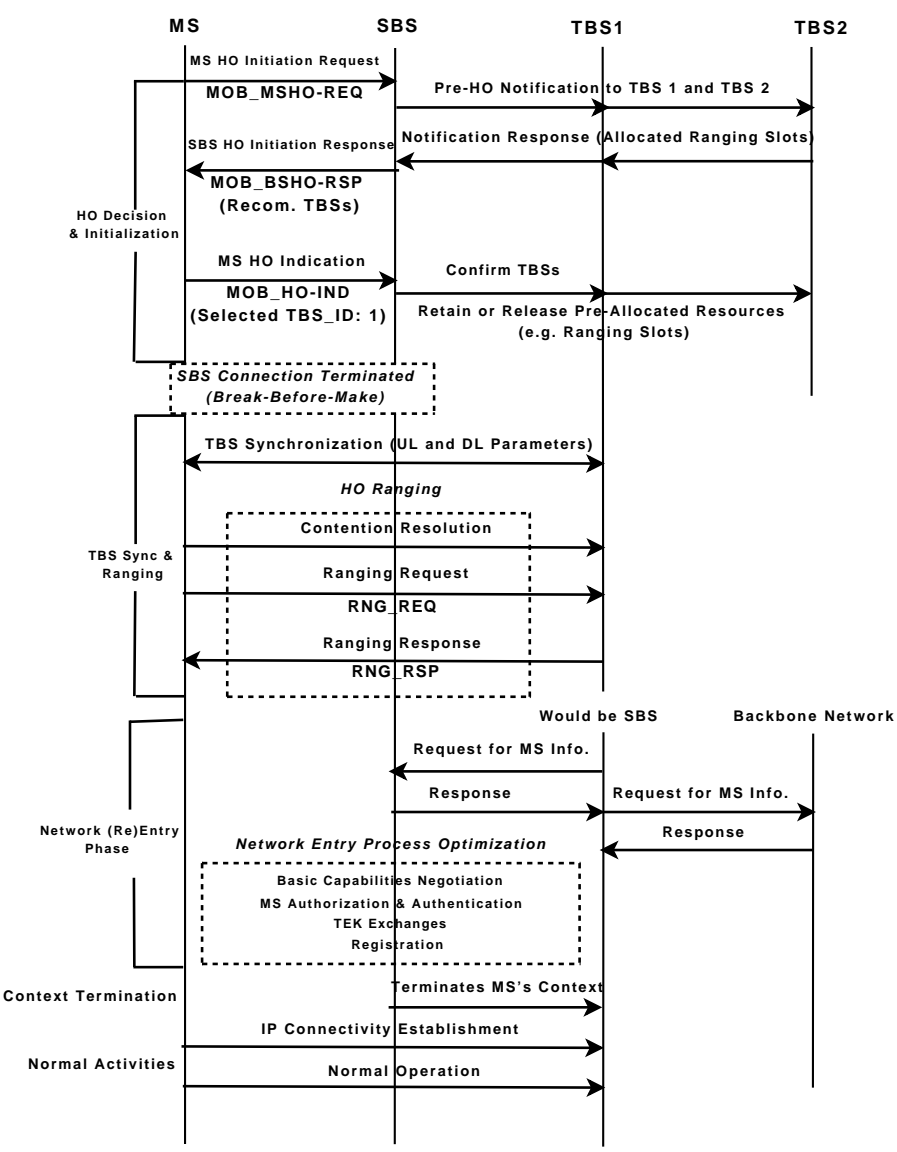

Fig. 3. AHOP Message Sequence Charts

its DS and ABS. The important concepts in the MDHO and FBSS approaches are:

- Diversity Set Updating: Update of the DS at any time depends on two different thresholds, the H_Add threshold and the H_Delete threshold, contained in the Downlink Channel Descriptors (DCD) that are broadcasted by the BSs. Based on a given MS's scanning of the BSs, those active BSs in its current DS with long-term CINR lower than the H_Delete Threshold value are deleted from the current DS and new active BSs with long-term CINR more than the H_Add Threshold value are inserted in the current DS.

- Updating and Selecting the new ABS: Update and selection of the new ABS for the modified DS is done by its MS and the BSs based on the signal strength measurements performed. For doing this, 802.16e uses either the traditional MAC Management mechanism or the Fast ABS Selection Feedback mechanism [2].

- Handover Occurrence: In both the MDHO and the FBSS mechanisms, a handover occurs when a new BS, having a more powerful signal strength than the serving BS, moves into the Active Set when it is updated. In the case of MDHO, during the handover, the MS simultaneously transmits or receives unicast messages and traffic from 
multiple BSs included in the DS. On the other hand, in FBSS, the normal handover procedure is not invoked while the MS switches BSs from the current ABS to the newly selected target ABS. The MS and the current ABS jointly do the selection of the target ABS [7]. During the BS switching, the MS remains connected to the current and the target ABSs.

\section{Comparative Advantages of the Handover Techniques in MWiMAX}

1) $\mathrm{HHO}$ : The HHO mechanism in the IEEE $802.16 \mathrm{e}$ is very similar to that used in Beyond 3G (B3G) technologies like EV-DO [8] and HSDPA [9]-[10]. However, unlike its cellular competitors, the HHO scheme in $802.16 \mathrm{e}$ is highly bandwidth efficient, fast, smooth and nearly glitch-free. This Network Optimized HHO mechanism [11] has the potential to minimize handover overheads and achieve a layer- 2 handover delay of less than $50 \mathrm{~ms}$ in the case of high-speed full mobility. This is the simplest MWiMAX handover technique ensuring efficient support for the provisioning of different high-speed real-time applications without significant interruptions and degradations of QoS. As in any other HHO technique, in MWiMAX too, an MS assumes that any new target BS always has adequate resources available to accommodate it, thus reducing the chances of call drops and delays.

The seamless nature of the HHO procedure in a typical MWiMAX sectorized deployment scenario facilitates lossless inter-frequency handover between sectors having different carrier frequencies but a fixed frequency reuse pattern [12]. The MWiMAX PHY and MAC layers provide support for dynamic and correct measurements of UL and DL signal strengths of the NBSs by the MS and the SBS, as well as efficient support for broadcast-related features. This helps to lower resource wastages and handover delays. However, the real advantage of MWiMAX's HHO scheme is the low deployment cost of the HHO, requiring very few spaced apart BSs.

2) MDHO And FBSS: The MWiMAX HHO model is not very attractive for handling voice-centric applications with high-speed mobility users. On the other hand, the two optional handover procedures MDHO and FBSS are designed to allow full seamless mobility at much higher speeds (up to 120 $\mathrm{kmph}$ ). With design features allowing very low (less than $1 \%$ ) or almost zero packet loss, very fast switching and low handover latency (less than $50 \mathrm{~ms}$ ), these two inter-sector handover techniques have all the potential to support highspeed real-time voice-centric applications like VoIP. Of course, to achieve this, the deployment cost would be considerably greater compared to the $\mathrm{HHO}$ model, as a larger number of MWiMAX BSs would be required within a specified area.

In a MWiMAX scenario, both MDHO and FBSS models have the capability to further reduce the handover delays and save more resources, as these two techniques do not require invocations of explicit $\mathrm{HO}$ signaling messages [2] when switching ABSs within the current AS. Moreover, their network re-entry procedures need not be performed every
TABLE I

BRIEF COMPARISON OF THE MWIMAX HANDOVER TECHNIQUES

\begin{tabular}{|l||l||l||l|}
\hline Parameters & Hard Handover & FBSS & MDHO \\
\hline Latency & High & Medium & Low \\
\hline Complexity & Low & Medium & High \\
\hline Reliability & Low & Medium & High \\
\hline Packet Loss & High & Low & Low \\
\hline Cost & Low & Medium & High \\
\hline $\begin{array}{l}\text { Support for De- } \\
\text { lay Sensitive Ap- } \\
\text { plications }\end{array}$ & Low & High & High \\
\hline Speed & Low & Medium & High \\
\hline Link Quality & Low & Medium & High \\
\hline
\end{tabular}

time when switching of anchor BSs is done. Further, unlike HHO, both MDHO and the FBSS have the advantage of performing handovers within sectors having the same carrier frequency, due to their employing the universal reuse concept [12]. However, between the two, owing to provision of better support for handling delay-sensitive applications, FBSS is the preferred handover option in such cases.

Both the macro-diversity handover schemes used in MWiMAX are designed to provide better performance with respect to multi-access interference, flexibility and coverage, than their CDMA competitors do. Application of both OFDMA fully used sub-channelization (FUSC) and partially used sub-channelization (PUSC) techniques in MWiMAX [12] macro-diversity handover mechanisms has improved the range and cell coverage. Much research activity in this area is being carried out globally by organizations like Intel, Nortel, Alvarion and others, with the aim of further improving the coverage, particularly at the cell edges. Another advantage of MWiMAX MDHO and FBSS is the ability of these techniques to enhance the ultimate system capacity. Depending on the underlying radio-link conditions, an MS can dynamically activate and deactivate these when required, to prevent unnecessary wastage of radio resources [13].

Finally, it can be concluded that, though both MDHO and FBSS offer significantly better handover performance in comparison to $\mathrm{HHO}$, there is still a long way to go before adequate support measures for these two techniques can be developed and deployed in MWiMAX networks. Accurately sharing the same carrier frequency among the multiple BSs in the AS, perfectly synchronizing the active set BSs and handling the increased deployment expenses, seem to be the major challenges so far. Table I provides a brief comparison of the three handover techniques with respect to the MWiMAX HO scenario.

\section{MWiMAX Deployment ARChitectures}

Currently the Network Working Group (NWG) in the MWiMAX forum is working on the implementation of a full-fledged MWiMAX mobility architecture supporting both homogeneous and heterogeneous mobility. However, devising a successful mobility and handover management framework depends much on the choice of suitable network deployment 
architecture. While a hierarchical or centralized architecture of $3 \mathrm{G}$ networks is suitable for supporting high-speed user mobility, it suffers from high latency and high cost [4]. On the other hand, low latency flat architectures, as in recent Wi-Fi networks, do not really support high-speed mobility. Although nothing has been decided yet, this alternative is apparently more suitable for Layer 3 implementation (which is yet to be standardized), as the different MIPv6 functionalities can be implemented without taking the facts and facets of the underlying technological implementations much into consideration, The NWG is currently deciding on the best Layer 3 implementation protocol deployment architecture to meet all the above objectives. A brief discussion on the different potential MWiMAX deployment architectures is presented here in order to help the reader understand how the different layers and the issues are related to these architectures.

Figure 4 shows three probable MWiMAX deployment architectures consisting of multiple subnets with individual characteristics. In Figure 4(a), which shows a MWiMAX centralized architecture, a subnet consists of one Access Network Gateway (ASN_GW) and multiple BSs under its control. The ASN_GW has centralized control of the subnet. The IP-layer functionalities are also located in the individual ASN_GWs, which efficiently support seamless handover along with low latency micro and macro-mobility activities. In contrast, Figure 4(b) shows the flat architecture, an alternative deployment scenario. In this case, a subnet consists of exactly one BS and one ASN_GW. The IP-layer functionalities are located in the individual BSs. The architecture supports macro-mobility and handover with optional session anchoring capabilities [4]. A third option may be the hybrid architecture (Figure 4(c)) in which different $\mathrm{BSs}$ control the handover and radio resource activities. In this context, we will explain ASN-anchored mobility and $\mathrm{CSN}$-anchored mobility, respectively, with respect to layer 2 and layer 3 handovers in MWiMAX for a better understanding of different situations in those layers.

\section{MWiMAX AND LTE: A BRIEF COMPARATIVE STUdy of Mobility And Handover Aspects}

Before long, telecommunication companies and operators worldwide will have a tough time in deciding on which access technology to choose for their consumers. MWiMAX could face strong challenges from some of the near-future technologies like 802.11n [14], 802.20 [15], iBurst [16] and LTE [5]. IEEE $802.11 \mathrm{n}$ is expected to get standardized by the end of 2009. However, the recent amendments made in the draft are apparently facing some problems with the Commonwealth Scientific and Industrial Research Organization (CSIRO) of Australia, which owns a few of the patents used in the draft [14]. This might deter some vendors from taking up the technology. The IEEE 802.20 standard, originally harnessed within the 16e working group and optimized for long-range wireless broadband mobility of data, has much in common with 802.16e. Therefore, it is unlikely that vendors already planning to push the $802.16 \mathrm{e}$ technology would again be interested in adopting the 802.20 in future. Moving to iBurst, a HighCapacity Spatial Division Multiple Access-based technology developed by ArrayComm and heavily backed by a leading manufacturer, Kyocera, offers full-mobility handovers but at a higher cost than MWiMAX. Lastly, the 3GPP LTE, which is expected to hit the market sometime in 2010, is forecasted by analysts as the 3GPP's response to MWiMAX, in order to be in the forefront of the wireless communication market. So, 3GPP LTE can be considered to be the strongest potential competitor to MWiMAX technology. Below we present a brief comparison of the mobility and handover aspects of LTE and MWiMAX.

The main drivers of the B3G LTE technology are better coverage, higher throughput, increased capacity and weaker latency requirements. The LTE architecture shown in Figure IV consists of BSs called eNBs, which are interconnected by the X2 links. The eNBs are connected to the Mobility Management Entity (MME)/SAE Gateway by the S1 links. Unlike in MWiMAX, the eNBs can directly communicate with each other and make intra-LTE handover decisions independently. Also, LTE is aimed at providing full mobility in the range of $350-500 \mathrm{~km} / \mathrm{h}$ and global roaming. Macrodiversity soft handovers are not supported by LTE. Table II compares the mobility and handover-related features of these two technologies.

It shows that 3GPP is projecting LTE as being more powerful than the existing versions of MWiMAX. Of course, LTE will face a strong challenge from the future 802.16m [17] version of MWiMAX, which is targeted for standardization towards the end of 2009 [18]. The major drawback of LTE in comparison to MWiMAX is its delayed commercialisation, which is planned for 2011 in the earliest. However, global telecommunication analysts are optimistic in predicting that the two technologies will converge rather than become competitive. This is because, being increasingly based on a similar set of telecommunication technologies, both have the capabilities to deliver higher mobility, greater bandwidth, larger range and flexibility with handover options. While the $802.16 \mathrm{~m}$ version is adopting many features of LTE, the latter will also use solutions similar to those of the existing and future mobility versions of MWiMAX. Hence, it is expected that both technologies would have increasing overlap in future markets. Convergence would occur not only in handheld multimode user devices and in laptops, but also in providing seamless session handover capabilities between the two while roaming (both being IP-based). Moreover, somewhat similar architectures would make it easier to provide seamless support for IPTV, VoIP and other Session Initiation Protocol-based services even while roaming.

\section{RESEARCH ISSUES IN MWIMAX HANDOVER SCENARIOS}

Any new technology faces many technological and nontechnological hurdles and challenges at its early stages and broadband MWiMAX is no exception. Despite significant volume of research activities going on worldwide, universally accepted efficient MWiMAX location and handover management frameworks are yet to be developed. This is in contrast to the cellular-based technologies that have got many years of 


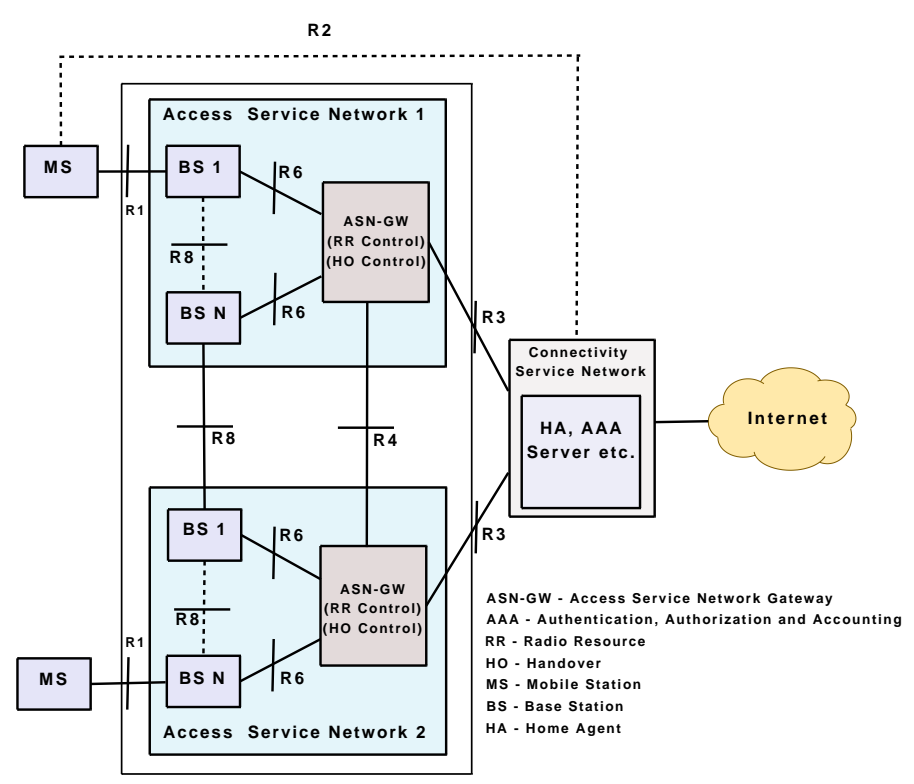

(a) Centralized

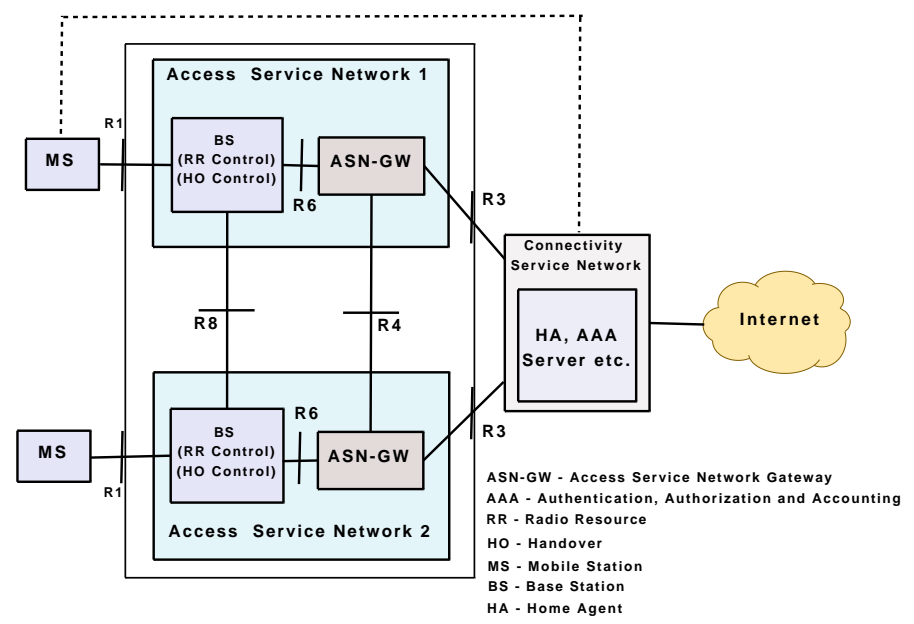

(b) Flat

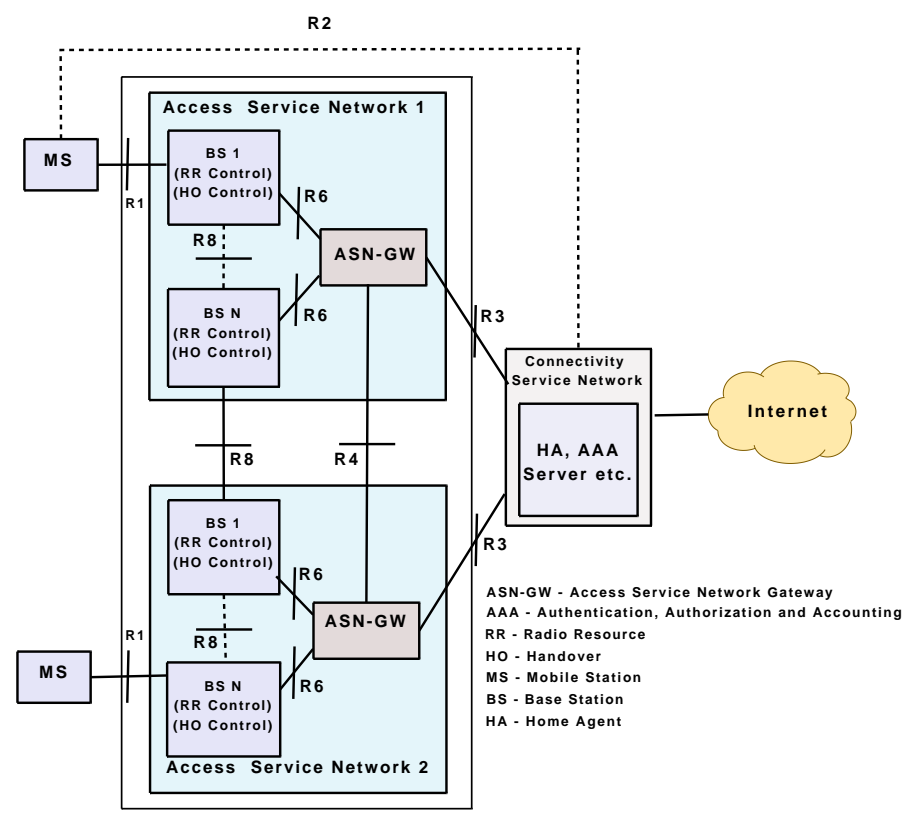

(c) Hybrid

Fig. 4. MWiMAX Deployment Architectures: (a) Centralised [12] (b) Flat [12] (c) Hybrid 
TABLE II

MoBiLITy AND HANDOVER-RELATEd COMPARISON BETWEEN MWiMAX AND LTE

\begin{tabular}{|c|c|c|}
\hline Parameters & MWiMAX & LTE \\
\hline HO Types Supported & Both $\mathrm{HHO}$ and SHO & No MDHO (SHO) \\
\hline Mobility & $\begin{array}{l}\text { Limited and Nomadic Mobility (up to } 120 \mathrm{~km} / \mathrm{h}) \text { : } \\
802.16 \mathrm{e} \text {; Full Mobility }(350 \mathrm{~km} / \mathrm{h}): 802.16 \mathrm{~m}\end{array}$ & Full Mobility $(350-500 \mathrm{~km} / \mathrm{h})$ \\
\hline Network Architecture & Centralized, Flat, Hybrid, IP-based; BS + ASN-GW & Very Flat, IP-based; eNB + MME/SAE GW \\
\hline Services & Packet Data and VoIP & $\begin{array}{l}\text { Packet Data and VoIP (more efficient for VoIP opti- } \\
\text { mization) }\end{array}$ \\
\hline Access Technology & SOFDMA in UL and DL (for 802.16e) & DL: OFDMA; UL: SC-FDMA \\
\hline Expected HO Latency & 35-50 ms: $802.16 \mathrm{e} ;<30 \mathrm{~ms}: 802.16 \mathrm{~m}$ & $<50 \mathrm{~ms}$ \\
\hline Backwards Compatibility & None Still & Full 3GPP Interoperability \\
\hline Roaming Supported & MWiMAX - MWiMAX (i.e. local / regional) & Full Global Roaming \\
\hline $\begin{array}{l}\text { Cell Radius (during mo- } \\
\text { bility) }\end{array}$ & $2-7 \mathrm{~km}$ & $5 \mathrm{~km}$ \\
\hline HO Decisions Depends & On MS and SBS & On eNB \\
\hline
\end{tabular}

IP CORE NETWORK

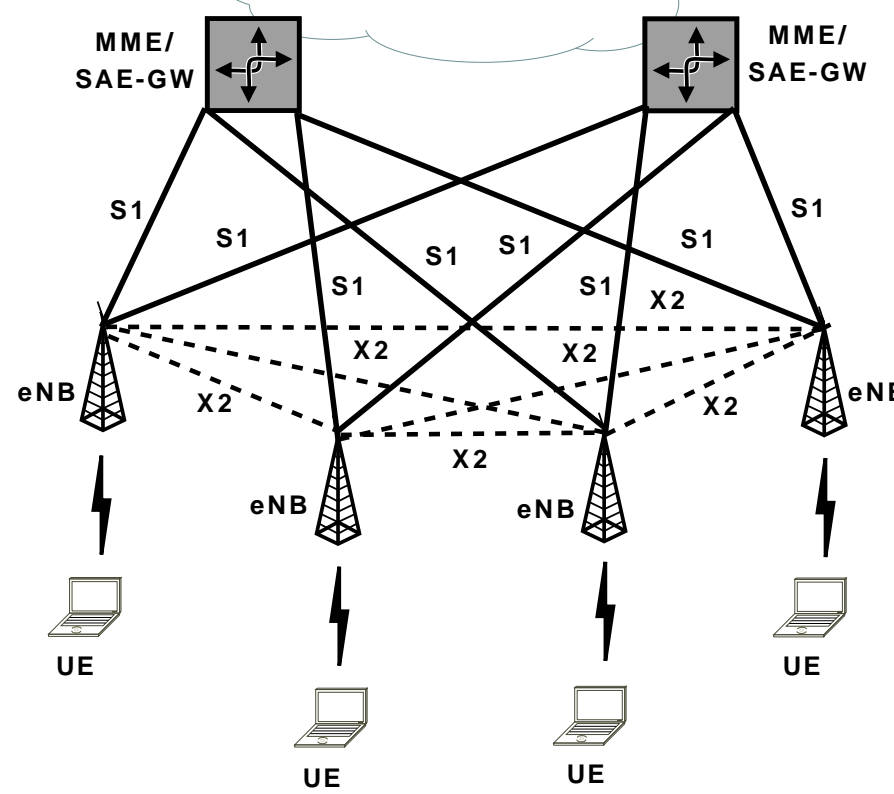

Fig. 5. LTE Architecture

experience in providing mobility support to users. Though the IEEE group dealing with the MWiMAX family of standards has come up with HHO, MDHO and FBSS techniques to deal with all types of applications, these procedures are not free from their own technical drawbacks. Figure 6 gives a concise overview of some of the probable layer 2 (L2), layer 3 (L3) and cross-layer (L2+L3) research hurdles that may hinder the successful design and implementation of a globally accepted MWiMAX handover management framework. In this section, these highlighted issues will be discussed in detail.

\section{A. MWiMAX Layer 2 Handover Issues}

In this case as shown in Figure 4(a), the BSs support only PHY and MAC-layer functionalities and any intra-subnet handovers (e.g. from BS1 to BSN within ASN1) are carried out using MAC-layer mobility management functionalities only. Such situations arise in the case of ASN-anchored mobility (intra-ASN mobility) where an MS's movement inside a subnet is controlled by the particular ASN-GW of that subnet. The individual ASN and MSs generally control all handovers in these cases, with support from the different BSs in the subnet. In case of layer 2 handovers, no change in the MS IP (network) layer configuration takes place. MWiMAX handover procedures, irrespective of the layered handover architecture, suffer from a huge range of issues, like resource wastage, high latency, unwanted packet losses, call drops and ping-pong activity, to name a few. Therefore, for each layer, new ideas have been proposed to deal with these and related problems. This section discusses the various MAC layer handover problems encountered by the HHO, FBSS and MDHO techniques in MWiMAX.

1) $\mathrm{HHO}$ Technique: Despite the fact that $\mathrm{HHO}$ is the mandated and the most bandwidth-efficient handover technique in MWiMAX, yet such handover activities are crippled by serious problems like excessive scanning activity in a somewhat non-optimized scanning interval before finalizing a TBS and prolonged inter-handover connection gaps. Though these issues are still drawing major research attention, as discussed below, several other important issues, such as unwanted network re-entry activities during the handover owing to ping-pong effects, IP connectivity delay during the network re-entry phase, and optimization of handover-based load distribution, have yet to be investigated in much depth. Apart from these, the subsections below also discusses less important HHO issues in a MWiMAX environment like efficiently exploiting both the UL and DL signals of the SBS and MS before initiating a handover activity and means of avoiding the wastage of unused ranging slots during pre-handover situation. A summary of these issues 


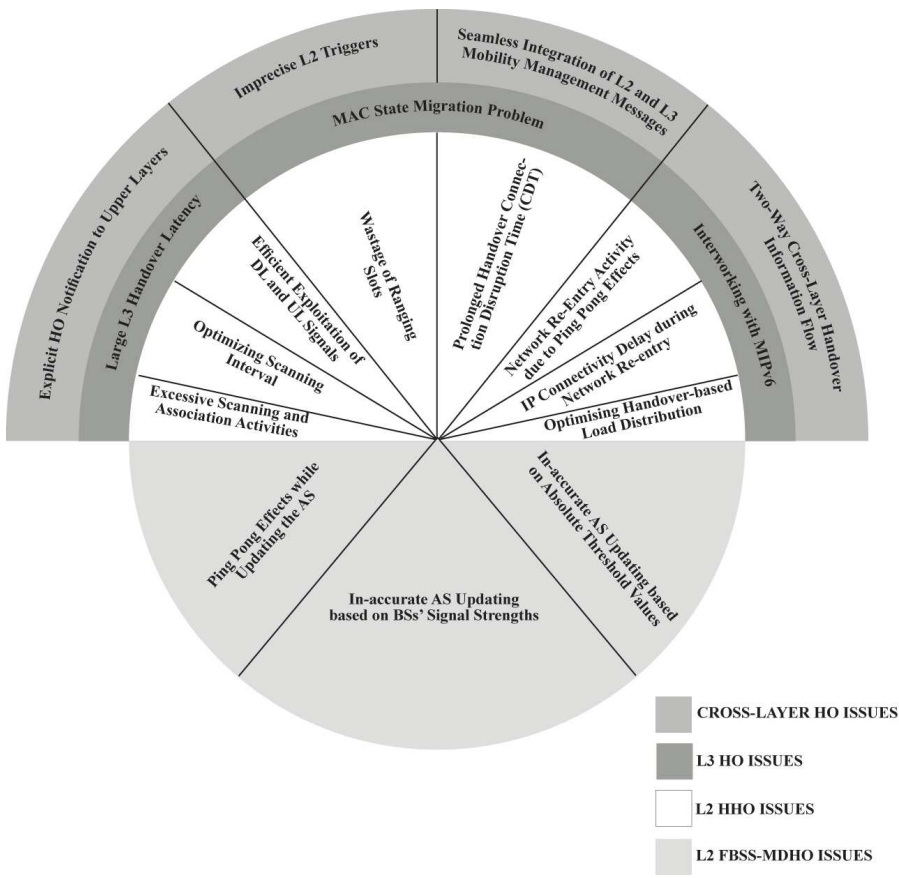

Fig. 6. MWiMAX Handover Research Issues

is provided in Table III to give the reader a better overview of the different aspects discussed before going into the details.

a) Excessive Scanning and Association Activities: One of the primary advantages of MWiMAX handover techniques is the provision of both layer 2 (L2) broadcast and scanning concepts during the NTAP by which the MS can receive channel signal strength information of its NBSs. The MS can scan some of the NBSs as potential TBS candidates. However, the $\mathrm{HO}$ technique does not clearly say anything regarding the number of NBSs that a MS may need to scan before ultimately deciding a TBS. This may result in redundant scanning of NBSs [19] leading to unnecessary wastage of channel resources and degrading the overall performance. Moreover, along with scanning, synchronization, ranging and association activities are also performed one after another (i.e. not simultaneously) during the NTAP. Hence, redundant scanning, and followed by prolonged synchronisation, ranging, and association activities proportional to the number of NBSs scanned, increases the overall handover delay. Also, while excessive scanning of the NBSs may affect the scheduler performance of the SBS particularly for the delay sensitive downlink traffic, unnecessary contention-based ranging results in unwanted consumption of the contention slots affecting the overall throughput [28].

Potential Research Solutions: A number of measures have been proposed to simplify scanning related procedures during the topology acquisition phase, to minimize the overall delay and enhance the system performance. The authors of [19]-[20] have proposed unique network topology acquisition schemes to identify the potential TBS before performing any type of scanning-related activities. In [19], the authors argued that, from the MOB_NBR-ADV messages, the MS can acquire the preamble-based mean Carrier to Interference-plus-Noise Ratio (CINR) along with the arrival time difference of the downlink signal (relative to the SBS) of the individual NBSs. From that, it can select the TBS to be the one having the biggest mean CINR and smallest arrival time difference. Then, the MS performs ranging, synchronization and association activities only with that TBS. Though this scheme reduces the handover delay by skipping unnecessary scanning, it considers neither the MS's direction of motion nor the current load of the selected BS. This might lead to unwanted ping-pong activity as well as call drops. In [20], it is proposed to predict the potential TBS prior to any scanning activity based on the different parameters like MS's movement direction, average time differences between previous handovers, position and distance of NBSs with respect to the SBS and load of the different NBSs. This scheme not only reduces the scanning-oriented overloads but also proves to be energy-efficient as the ranging procedure (which consumes lots of energy) is only limited to the particular predicted TBS. Another idea discussed in [29] is to modify the MOB_NBR-ADV broadcast message, which contains static channel-related information on the NBSs, to provide link quality parameters-oriented dynamic information on the BSs. This would decrease the need for scanning as the MS can gather more handover decision related information from broadcast messages themselves. Elimination of NBSs as TBS candidates, prior to scanning, depending on QoS, active service flows and bandwidth requirements of the MS, is also a good solution for avoiding unwanted scanning activities [30]-[31]. However, in spite of all these proposals, there is still a need to come up with universally accepted ideas regarding dealing with unwanted delays and wastage of channel resources owing to excessive scanning, ranging and association related activities during MWiMAX handover operations. Standard means for performing the CINR measurements are also desirable.

b) Optimizing Scanning Interval: In the MWiMAX HHO scenario, scanning of multiple channels is an inevitable activity for discovering the NBS, which is most suitable to be the potential TBS. Hence, though it is difficult to avoid scanning process completely, one can try to keep it within limits, as discussed previously. During scanning, MWiMAX handover mechanisms temporarily pause the uplink and downlink of data transfer between the MS and the SBS. These scanning intervals are allocated by the SBS dynamically on getting scanning interval allocation requests from the MS. However, frequent temporary suspension of data exchange lowers the system throughput, and adds more delays to the overall handover process. Also, QoS requirements may get disrupted owing to this. Moreover, during scanning intervals, all data meant for the MS are buffered at the SBS, what leads to wastage of channel resources. Hence, it is desirable to devise techniques of effective estimation and minimisation of both the frequency and the time interval needed for scanning. Required also are the methodologies to carrying out scanning and data exchange concurrently. 
TABLE III

Summary of THE Probable MAC-LAYER HHO-RELATEd ISSUES IN MWiMAX

\begin{tabular}{|c|c|c|}
\hline Issues & Effects & Proposed Research Directions \\
\hline $\begin{array}{l}\text { Excessive Scanning and } \\
\text { Association Activities }\end{array}$ & $\begin{array}{l}\text { Redundant NBS scanning, ranging and association } \\
\text { activities may lead to unnecessary L2 handover delay } \\
\text { and resource wastages. }\end{array}$ & $\begin{array}{l}\text { Based on parameters like MS's trajectory of motion } \\
\text { and previous HO intervals along with link quality } \\
\text { information [19]-[20] of the NBSs, an MS can select } \\
\text { the potential TBS before the scanning operations. }\end{array}$ \\
\hline $\begin{array}{l}\text { Optimizing Scanning In- } \\
\text { terval }\end{array}$ & $\begin{array}{l}\text { Temporary suspension of data exchange between the } \\
\text { MS and the SBS during scanning interval degrades } \\
\text { the overall handover performance. }\end{array}$ & $\begin{array}{l}\text { In a multi-MS MWiMAX environment, NBSs can } \\
\text { exchange configuration parameters to figure out the } \\
\text { ideal scanning interval required [21]. }\end{array}$ \\
\hline $\begin{array}{l}\text { Efficient Exploitation of } \\
\text { DL and UL Signals }\end{array}$ & $\begin{array}{l}\text { QoS may be hampered if both downlink and uplink } \\
\text { parameters are not considered during handover initi- } \\
\text { ation and execution. }\end{array}$ & $\begin{array}{l}\text { Combination of effective measurements of MS's } \\
\text { uplink signal strengths and SBS's downlink signal } \\
\text { strengths at the handover region enhances the han- } \\
\text { dover performance [22]. }\end{array}$ \\
\hline Wastage of Ranging Slots & $\begin{array}{l}\text { The non-retained ranging slots of the other candidate } \\
\text { BSs, allocated during the scanning phase, add up to } \\
\text { the handover resource wastage after the MS selects } \\
\text { the particular TBS [19]. }\end{array}$ & $\begin{array}{l}\text { Selection of the TBS prior to the handover pre- } \\
\text { registration phase [19]-[20] can debar other candi- } \\
\text { date BSs from allocating ranging slots. }\end{array}$ \\
\hline $\begin{array}{l}\text { Prolonged Handover Con- } \\
\text { nection Disruption Time } \\
\text { (CDT) }\end{array}$ & $\begin{array}{l}\text { Inter-handover connection gap degrades QoS owing } \\
\text { to service disruptions. }\end{array}$ & $\begin{array}{l}\text { New MAC management message [23] can enable the } \\
\text { MS to receive traffic immediately after the handover. } \\
\text { Also, MS can perform the new network entry process } \\
\text { during its idle period to receive traffic continuously } \\
\text { [24]. }\end{array}$ \\
\hline $\begin{array}{l}\text { Network Re-Entry Activ- } \\
\text { ity due to Ping Pong Ef- } \\
\text { fects }\end{array}$ & $\begin{array}{l}\text { Unnecessary network re-entry procedures owing to } \\
\text { ping-pong effects cause delays and call disruptions. }\end{array}$ & $\begin{array}{l}\text { The SBS notifies the MS about the time duration that } \\
\text { the traffic for MS will remain buffered in the SBS } \\
\text { [25]. This avoids network re-entry procedures. }\end{array}$ \\
\hline $\begin{array}{l}\text { IP Connectivity Delay } \\
\text { during Network Re-entry }\end{array}$ & $\begin{array}{l}\text { MS needs to know more clearly during or before } \\
\text { the network re-entry activity whether a switch in the } \\
\text { IP connectivity is required after the HO. Otherwise } \\
\text { unnecessary connectivity activities only enhance the } \\
\text { overall delay. }\end{array}$ & $\begin{array}{l}\text { If the TBS can know of the MS's previous AR and } \\
\text { the IP address, it can help in reacquiring the MS's } \\
\text { IP connectivity context [26] }\end{array}$ \\
\hline $\begin{array}{l}\text { Optimising Handover- } \\
\text { based Load Distribution }\end{array}$ & $\begin{array}{l}\text { Evenly balancing the traffic loads and evenly dis- } \\
\text { tributing available resources over different BSs in an } \\
\text { area is important in MWiMAX. Solving this issue } \\
\text { would not only enable better QoS but would also } \\
\text { weaken call disruptions and call blockings. }\end{array}$ & $\begin{array}{l}\text { Both BS-initiated directed handovers and MS- } \\
\text { initiated rescue handovers are conducted in parallel } \\
\text { to offer better load balancing scheme enabling satis- } \\
\text { factory QoS and much fewer ping-pong effects [27] }\end{array}$ \\
\hline
\end{tabular}

Potential Research Solutions: It should be noted that, as the QoS might get hampered in case of both long and short scanning intervals, optimisation of scanning intervals is an important issue. An efficient Adaptive Channel Scanning algorithm in a multi-MS oriented MWiMAX environment, relying on the exchange of configuration parameters between the NBSs in order to find out the required scanning time for a MS, is proposed in [21]. Along with optimisation of the allocated scanning intervals for all MSs, the scheme also maintains the QoS of the application traffic in the system. However, utilization of unlimited channel buffers, in order to make the packet loss almost negligible, complicates the problem of channel resource wastage. Another proposal, for minimizing the influence of scanning intervals by concurrent scanning and data transmission by the MS is discussed in [19]. This fast synchronization and association model uses the unique IDs of the SBS and the NBSs (unique BSIDs), to distinguish between the UL/DL messages of the SBS and the NBSs. As the MS can clearly identify and separate the SBS's data exchange messages from the NBSs' synchronization and association messages, it can communicate to both of them at the same time, with the ranging slots appropriately adjusted by the SBS to minimize the chances of collisions. This scheme, however, neither considers a multi-MS environment nor considers an environment where the different NBSs and the SBS might not be controlled by the same service provider network [21]. An MS's sleep mode option [2] also provides an interesting mechanism for the MS to perform scanning without hampering the transmission with the SBS.

\section{c) Efficient Exploitation of DL and UL Signals:}

MWiMAX promises to deliver streaming multimedia applications in the form of voice and data. However, the QoS of data and voice services might not be the same and their requirements may vary for UL and DL transmissions. This would degrade the system performance. Hence, to provide effective and stable QoS for all types of applications, it is advantageous to consider both UL and DL signal parameters while initiating and executing handover. This is particularly important for delay-sensitive voice and dataoriented applications in MWiMAX.

Potential Research Solutions: In a mobility scenario, the UL and DL signals of an MS and the SBS respectively are not strictly correlated with respect to distance between them. From an user's perspective, though, it seems that, as the distance between an MS and its SBS changes, the MS's UL signal strength measured at the SBS and the SBS's DL signal strength measured at the MS also changes in 
a correlated fashion, this is not true always. DL and UL signals are considered jointly in [22], to propose a hard handover scheme based on the MS's UL signal strengths and the SBS's DL signal strengths measured at the SBS and the MS, respectively. A handover process is triggered once the two signal strengths fall below some pre-determined thresholds. This scheme assumes that an MS does not need to perform unnecessary monitoring and scanning of the NBSs' signal strengths at the non-handover region in a cell before a handover is initiated. Unwanted delays as well as ping-pong and outage probabilities are reduced significantly. Though much work has not been done yet on utilizing both downlink and uplink signals to direct and initiate a MWiMAX handover, in comparison to the downlink signal-based schemes, this choice may have the potential to provide better QoS, reduced scanning requirements and improved overall system throughput. Clearly, it demands further research.

d) Wastage of Ranging Slots: MWiMAX supports handovers initiated by either the MS, or the SBS, or even the underlying network. In case of MS-initiated handovers, when the suitability of the potential candidate NBSs selected by the MS during the NTAP is accepted, the individual BSs allocate ranging slots for the MS, which then selects the new TBS and retains only the ranging slots provided by that BS. The other unused ranging slots add up to the list of resources being wasted during the entire handover process.

Potential Research Solutions: Such wastage of unwanted resources can be avoided if the SBS can select the new TBS before the allocation of ranging slots, as proposed in schemes [19]-[20]. So, once selected, only that TBS may allocate ranging slots, debarring the other NBSs from unnecessarily allocating such slots as well.

e) Prolonged Handover Connection Disruption Time $(C D T)$ : Being a 'break before make' technique, the HHO concept in MWiMAX suffers from a lengthy "inter-handover" CDT that could lead to unwanted hazards like packet losses, call disruptions or even call drops, while on the move. This occurs in the actual handover phase, when an MS terminates the connection with the SBS and tries to set-up connections with the selected TBS. While a CDT in the range of $200 \mathrm{~ms}$ is acceptable for real-time streaming media traffic [32], anything more than that is disruptive [33]. In MWiMAX, data, voice and multimedia contents are intermixed and each requires different mechanisms for its transmission, particularly during handover. So, such a lengthy CDT may cause serious service disruptions in case of real-time high-speed delay-sensitive voice and streaming multimedia applications in MWiMAX networks.

Potential Research Solutions: To counter the above drawbacks, considerable quantity of research work has taken place over the last few years to minimize the inter-handover service interval time. The IEEE MWiMAX group has incorporated the MDHO and FBSS techniques, which are ideal for delay sensitive applications like VoIP. However, as these two techniques are much complicated and can increase deployment costs, research activities have been carried out to further reduce the QoS related hazards during real-time services caused by the CDT.

Sik Choi et. al. [23] have proposed a link-layer fast handover scheme for MWiMAX HHO scenario that significantly reduces the probabilities of packet loss and transmission delay during handover. This scheme introduces Fast DL_MAP_IE MAC management message, which enables an MS to receive downlink traffic just after the downlink synchronization with the TBS, even before the completion of the uplink synchronisation phase. A similar idea, called Passport Handover, is discussed in [33] where an MS could resume the DL re-transmissions with the TBS before the completion of the authorization procedures, by using the CIDs of the previous SBS. Though both these mechanisms managed to achieve an improvement of the overall handover performance, they did not consider potential possibilities of unsuccessful authorization activities while switching domains. This is fixed in [34], in which having predicted the TBS by considering criteria like MS's movement trajectory, NBSs' locations and MS's average inter-handover gap, the SBS passes on MS's authorization parameters to the TBS over the backhaul network. Also, the ranging results are stored by both the TBS and the MS for a certain period of time until they are re-used during the connectivity disruption stage. Hence, this omits the need for a second ranging activity and the MS could thus switch domains very quickly without having to worry about authorization activities, which are already done during the pre-handover stage. However, there is still scope for research on these aspects, to see how smoothly the lengthy authorization approach could be done prior to the actual handover phase with or without the help of the backhaul network. This is because transferring the stored authorization messages from the SBS to the TBS may sometime increase the overall load in the backhaul network.

Another interesting idea proposed in [35] deals with an MS maintaining simultaneous network connectivity with the SBS and the TBS. In this case, it is assumed that the coverage areas of the two BSs overlap so that the MS gets sufficient time to complete the network re-entry process at the target network, before it looses the connectivity with the SBS. This may be a possible scenario in the case of MWiMAX networks due to the large coverage areas of the BSs. However, this scheme requires further study to investigate such feasibility factors as duration of overlap, effects of blind spots at the overlapped regions and the cost. MS's idle periods could also play an important factor in this issue as suggested in [24]. As stated there, if the MS performs the network re-entry signaling with the TBS during the idle mode of the MS, it would allow the MS to continue data exchange simultaneously with the SBS leading to a very low latency HO procedure. However, this idea requires the BSs to be synchronized, and this might be a problem in case of HHO. Therefore, it still remains a research challenge to devise suitable frameworks for dealing with the CDT issue in MWiMAX HHO. 
f) Network Re-Entry Activity due to Ping-Pong Effects: In MWiMAX HHO, when an MS wants to get connected to a new BS, it has to complete the entire network re-entry procedure comprising of the series of security and connection re-establishment processes. This takes a long time. In a situation where in the middle of an ongoing communication, an MS, that is performing network re-entry procedures with a TBS, wants to come back to the previous SBS due to change in signal strengths, it leads to further delays if the entire re-entry procedure needed to be performed again for the old SBS. Handover overheads caused by unnecessary re-entry procedures resulting from such ping-pong effects may degrade the overall system performance.

Potential Research Solutions: What really needed is to devise mechanisms to make the previous SBS able to differentiate ping-pong re-entries from new re-entries, so that overall re-entry phases for the previous one could be shortened. Research carried on this problem resulted in a mechanism in which the TBS, upon learning about the ping-pong effect, intimates the previous SBS about the MS's reverting back to it [36]. This will help the previous SBS to identify the return of the MS as an effect of ping-pong and not as a new network entry altogether. So, not only will it provide non-contentious ranging opportunities to the returning MS, but will also resume the communication quickly, provided the SBS has retained the MS's connection information. However, this scheme will not work if the SBS has not retained the state information of the MS. In that case, however, the allocated ranging slots for the returning BS will be wasted. So, a more authentic method is proposed in [25] in which, prior to a handover, the SBS intimates to the MS about how long the MS's connection information would be retained. During the ping-pong effect, if the MS knows that the SBS is still retaining the previous connection information, it can act accordingly to quickly resume the previous communication with the SBS. Also, in case of a dropped call during handover, the TBS can use the connection information retained by the SBS regarding the MS and can very quickly perform the call recovery procedure. However, there is no suitable explanation for such a scenario when an MS, due to the ping-pong effect, has to come back to the SBS in spite of knowing that the SBS is not retaining the previous connection information any longer. Further research is needed to deal with such situations arising from the ping-pong effect. Minimization of handover overheads, reduction of resource wastages and early recovery of any call drops are the important factors, which should be kept in mind while formulating such solutions.

g) IP Connectivity Delay during Network Re-entry: During a MWiMAX HO process, if an MS moves to a TBS under the same access router within the same subnet, then the HO does not incur any change in the MS's IP connectivity scenario. MS's IP connectivity context with reference to the new SBS remains the same as with the old SBS. However, this is not the case if the TBS falls under a different subnet altogether. In that case, the MS has to go for the lengthy procedure of IP connectivity acquisition during the re-entry phase to complete the $\mathrm{HO}$ process. In the current scenario, it is clearly a challenging issues of how an MS actually determines whether a change in the IP connectivity context is at all required as part of an ongoing $\mathrm{HO}$ activity. If a change is not required then it would save significant amount of HO-related latency as the MS would not go for that at all. In the current MWiMAX standard, a $\mathrm{HO}$ optimization flag in the MOB_NBR-ADV message [2] indicates whether an IP subnet switch is required during a $\mathrm{HO}$ activity. However, this is not a very fruitful detection mechanism as it incurs administrative overhead.

Potential Research Solutions: In order to get rid of such delays, MSs need to figure out, beforehand, if the TBS falls under a different subnet altogether. If yes, then only it has to initiate the lengthy IP context acquisition procedure during the network re-entry phase, else not. A solution to this problem is proposed in [26]. Depending on the information provided by an MS, a TBS could reacquire the MS's IP connectivity context, thereby minimizing the overall delay. During a HO activity, the MS needs to provide the TBS information regarding its last IP address and Fully Qualified Domain Name (FQDN) of its last AR [26]. Based on these information, the TBS instructs the MS whether or not it can retain the previous IP connectivity contexts. Devoid of any administrative overheads, the solution claims to be independent of any MWiMAX RAN architecture.

h) Optimizing Handover-based Load Distribution: In a mobile communication environment, the QoS experienced by MSs can degrade significantly owing to increased traffic load in a cell. Problem like unbalanced traffic load distribution [37] between different adjacent cells can force the traffic load in a particular cell to exceed the ultimate capacity of that cell. With the overlapping nature of the cells, unevenly distributed resource utilizations among the different adjacent BSs incur additional cost and hamper the service quality. Therefore, evenly balancing the loads and evenly distributing the different available resources within a cluster of BSs is a relevant and interesting research issue. This is a problem in the MWiMAX scenario as well. Though the MWiMAX Forum has supported a Radio Resource Management (RRM) framework for efficient load balancing and resource utilization [38] with the help of BS-initiated directed handovers [27], the specification provides only a framework and lacks any detailed implementation concepts and algorithms [39]. Thus, it is an open research issue.

Potential Research Solutions: Here, MWiMAX research has been mostly focussed on designing and implementing an efficient algorithm for evenly distributing MSs, which reside on the overlapping areas of the adjacent cells, among adjacent BSs. Another idea, which has not been advanced much yet, is to gather the resources to areas where majority of the traffic is located [39]. The MWiMAX Forum has looked at the former idea. In the BS-initiated directed handover scheme, the congested SBS forces the MS to handover to a non-congested TBS. This BS-controlled and initiated HO scheme offers 
good QoS in comparison to traditional MS-initiated rescue HO schemes, in which the load balancing logic resides in the MSs and the MS handovers to a less congested TBS whenever the signal strength drops below a threshold.

An efficient load balancing scheme is proposed in [27] in which directed and rescue $\mathrm{HO}$ mechanisms are conducted in parallel. The scheme uses Spare Capacity Reports (SCR) [38] broadcasted by the different BSs in an area to let their peers know of their load. Depending on such reports, the BSs classify their loading states as underloaded, balanced or overloaded. Directed HO to a TBS occurs in the case of underloaded conditions, whereas rescue $\mathrm{HO}$ takes place if the TBS is in balanced or overloaded states. This scheme offers satisfactory QoS and much reduced ping-pong activities. Additionally, one could consider different prioritization means by which the MSs can be handed over to the TBS. They could take into account e.g. traffic priority and channel conditions [27].

Another proposal made in [40] considers an MS-initiated rescue HO mechanism, in which handovers between the different frequency assignments (FA) (MWiMAX assigns multiple FAs to the different operators) take place. As opposed to the standard MWiMAX HO scenario, where no target FAs are indicated, this scheme not only introduces the concept of target FAs but also offers seamless HO from the crowded serving FA to the non-crowded target FAs. Despite such research attempts, considerable work is still needed before choosing the BS-initiated directed $\mathrm{HO}$ scheme over the traditional MS-initiated rescue scheme.

2) MDHO And FBSS: Similar to the HHO, these soft handover techniques for supporting inter-sector handovers also suffer from few drawbacks. As discussed previously, while the drawbacks of the NTAP also hold true for these handover techniques, both MDHO and FBSS suffer from performance hindrance challenges, specifically with the accuracy of updates of the active sets during the actual handover phase. Not much work has been done for dealing with these important issues and as such, they are open for future research contributions. A summary of these challenges are highlighted in table IV, before a detailed discussion is presented in the next sections.

a) Ping-Pong Effects While Updating the AS: In MDHO and FBSS, depending on the signal strengths of the BSs, an MS always maintains an AS of NBSs, comprising of the NBSs with the most powerful signal strength at that particular instance of time. The AS also contains the serving or anchor BS (ABS). The other NBSs remain in the set of probable candidate BSs (candidate set) for the active set. The MS always monitors these BSs to update the AS, depending on a threshold value. However, specific discussions are required to determine the acceptable threshold value at any particular instance, to avoid unnecessary updating of the AS.

Potential Research Solutions: The difference between the new threshold value and the existing value should be large enough to trigger the requirements for AS updating as there are always possibilities that due to a very low threshold value difference, NBSs from the candidate set may move in and out of the AS unnecessarily. Such enhanced ping-pong activities would not only make the AS updates meaningless, but also hike the resource consumption in regard to the required signaling [41], degrading the overall performance. So efficient methods of determining the right threshold values to update the AS are required to reduce such performance-hampering activities.

b) Inaccurate AS Updating based on the BSs' Signal Strengths: The FBSS and MDHO rely on the 'signal strength' of the NBSs as the sole basis for updating the AS. They take into account neither the path followed by the MS, nor the mobility of the MS. Relying only on signal strengths does not always result in optimum performance, especially in regard to channel and resource wastages. This is because, in such cases, it can be concluded that the AS, at any particular instance, may get populated by such NBSs with which the MS will not perform a handover activity at the near future. Though the signal strengths of such NBSs may be strong enough to be included in the AS, they might not fall into MS's movement trajectory. Automatically such BSs would pop out of the AS after some time, when the MS moves further away from them, resulting in frequent and unnecessary updating of the AS. Thus, in terms of channel usage, inclusion of such NBSs is a complete waste.

Potential Research Solutions: Inclusion of unnecessary NBSs in the AS can be avoided if, along with the signal strengths, the MS also considers its direction of motion for choosing the AS constituents. The handover performance enhancing technique "Predictive Base Station Switching", for selecting and updating the current SBS and the AS at any instant, was proposed in [42]. This technique considers not only the signal strengths of BSs but also the current direction and speed of the MS, to make a selection decision from among the NBSs. The scheme also predicts the probable future behaviour of the MS while making a decision. It is thus imperative that future MWiMAX handover research on related issues, pay more attention to devising significant potential NBS selection techniques, taking into account the MS's direction of motion along with the NBSs' signal strengths. This will reduce unnecessary resource wastage and will result in a better system performance. However, the means of accurately estimating the speed of the MS and its direction of motion need to be formulated, especially during full vehicular mobility. Along with MS's movement trajectory, QoS requirements of the MS are also an issue. To provide the best network performance, AS should be updated with those NBSs that meet the QoS and bandwidth requirements of the MS.

c) Inaccurate AS Updating based on Absolute Threshold Values: In the MDHO and the FBSS, the MS updates the AS based on the absolute H_ADD and H_DELETE threshold values contained in the DCDs broadcasted by the BSs. At any instant, all the NBSs in the AS having 
TABLE IV

Summary of THE PRobable MAC-LAYER FBSS AND MDHO-RELATED ISSUES IN MWIMAX

\begin{tabular}{|l||l||l|}
\hline Issues & Effects & Proposed Research Directions \\
\hline $\begin{array}{l}\text { Ping Pong Effects while } \\
\text { Updating the AS }\end{array}$ & $\begin{array}{l}\text { Non-significant difference between new and existing } \\
\text { threshold values may cause unnecessary update of } \\
\text { the AS enhancing ping pong effects. }\end{array}$ & $\begin{array}{l}\text { Accurately analysing threshold values [41] reduces } \\
\text { unnecessary updating of ASs. }\end{array}$ \\
\hline $\begin{array}{l}\text { In-accurate AS Updating } \\
\text { based on BSs' Signal }\end{array}$ & $\begin{array}{l}\text { Channel resources may be wasted owing to inclusion } \\
\text { of unnecessary BSs in the AS depending only on } \\
\text { BS's signal strengths. }\end{array}$ & $\begin{array}{l}\text { AS upgrading process may also consider the MS's } \\
\text { direction of motion [42] along with the BS's signal } \\
\text { strengths. }\end{array}$ \\
\hline $\begin{array}{l}\text { In-accurate AS Updating } \\
\text { based on Absolute } \\
\text { Threshold Values }\end{array}$ & $\begin{array}{l}\text { Absolute threshold values may not be the best param- } \\
\text { eters to upgrade the AS in real-life situations where } \\
\text { load of cells changes dynamically. }\end{array}$ & $\begin{array}{l}\text { Relative threshold values can upgrade the ASs more } \\
\text { accurately [43]. }\end{array}$ \\
\hline
\end{tabular}

CINR value less than H_DELETE threshold are removed from set and those, from the candidate set (CS), with CINR values more than H_ADD threshold are added to the AS. However, in reality, with the load of a cell changing at every moment, relative threshold values instead of an absolute one seem to be more realistic for accurate updating of the AS.

Potential Research Solutions: A similar technique based on the relative threshold values was discussed in [43]. In this scheme, an NBS from the CS is transferred to the AS provided Neighbour_BS_CINR - ABS_CINR $<$ H_ADD threshold and a BS from the AS is transferred to the CS provided Active_BS_CINR - ABS_CINR $>$ H_DELETE threshold. Though this method provides a more accurate way of active set updating, yet it is more complicated to implement. Therefore, in the current day scenario, with a substantial increase in the number of mobile users each day, it is an uphill task to formulate suitable means of correctly choosing the threshold values at any particular instant of time in order to rightly update the AS.

\section{B. MWiMAX Layer 3 Handover Issues}

In MWiMAX technology, the network architecture from IPlayer onwards is still undefined and non-standardized. The IEEE MWiMAX group, after specifying the L2 HO-related over-the-air messaging and procedures, has left further designs and standardization of the architecture to the NWG of the MWiMAX Forum, which is currently developing the L3related network messaging and further $\mathrm{HO}$ procedures on top of the L2-base. Though several research activities are going on worldwide on designing a MWiMAX L3 onwards mobility management framework, its still a long way to go before something acceptable can be devised. A reference to the MWiMAX CSN-anchored mobility (inter-ASN mobility) is required here. A handover in such a macromobility scenario occurs when an MS moves from the current SBS in the current subnet to a different BS in a different subnet controlled by a different ASN-GW. Therefore, the IP-layer (L3) configuration of an MS changes as a result of such a handover. Unlike the ASN-anchored scenario, in this case the mobility management and the handover aspects engage both the ASN and CSN entities and are generally network-initiated [44]. Referring to Figure 7, whenever a terminal performs an inter-subnet handover (e.g. from BS1/BS2 under ASN-GW1 to BS3 under
ASN-GW2), it results to an IP-layer (L3) handover. It is related with re-configuration and reestablishment of new IPconnectivity. On the other hand, in Figure 4(b), every change of BS automatically implies a change in the subnet and thus a change in the IP-connectivity of a terminal. Hence, in such an architecture, the handovers always involve an alteration to the MS IP-layer configurations.

However, though the NWG of the MWiMAX Forum has embraced the different IETF protocols and provided a MWiMAX Network Reference Model (RFM) [28],[45] as a framework to develop the ASN and CSN-anchored mobility schemes, the technical solutions to the different handover-related issues discussed in this paper are left open for research on standard-compliant acceptable schemes and implementations. In this context, it should be noted that in comparison to the MAC-layer handover issues, those in the IP layer did not attract much research attention yet and as a new technology, with non-standardized network and upper layer architecture, MWiMAX faces a plethora of issues related to IP handover, starting right from large L3 handover latencies to suitable choices of handover protocols. These issues should not only be dealt individually but also along with L2 issues to get the optimum results. For example, in order to reduce the overall MWiMAX handover latency, it is required to reduce both the L3 handover latencies and the L2 handover latencies, in order to get the maximum reduction in latency. Hence, to do this, schemes should be devised not only to reduce the L3 handover latencies separately but also to tackle it jointly with the L2 handover latency. The L3 handover schemes in case of $\mathrm{CSN}$-anchored mobility are largely based on either MIPv4 or MIPv6, as in WLAN, but as MIP is not very suitable for providing sufficient handover performance of the different time-sensitive applications, hence both research community and the NWG are considering alternative means to tackle the L3 issues for designing effective MWiMAX inter ASN as well as ASN-CSN HO procedures in MWiMAX [46]. A summary (table V) is followed by detailed discussion on some of these issues, see subsections below.

d) Large L3 Handover Latency: Similar to WLAN and other cellular technologies, in MWiMAX too, during inter-subnet mobility, the overall handover latency is the sum of the handover latencies in the MAC and IP-layers. Compared to the L2 handover latency, the latency in the L3 
TABLE V

SUMMARY OF THE PROBABLE IP-LAYER HANDOVER ISSUES IN MWIMAX

\begin{tabular}{|l||l||l|}
\hline Issues & Effects & Proposed Research Directions \\
\hline $\begin{array}{l}\text { Large L3 Handover La- } \\
\text { tency }\end{array}$ & $\begin{array}{l}\text { Delay incurred in performing the different L3 han- } \\
\text { dover steps is large. This affects the overall handover } \\
\text { performance. }\end{array}$ & $\begin{array}{l}\text { Timely indication of organised L2 triggers [47]-[48] } \\
\text { can lead to early initiation of L3 handover activities. }\end{array}$ \\
\hline $\begin{array}{l}\text { MAC State Migration } \\
\text { Problem }\end{array}$ & $\begin{array}{l}\text { Non-transmitted MAC state frames during HHO may } \\
\text { be lost and the delay incurred in retransmitting them } \\
\text { may degrade the system performance. }\end{array}$ & $\begin{array}{l}\text { Serving network can buffer the IP packets meant for } \\
\text { the MS to reset the lost MAC frames from those } \\
\text { stored packets [12]. }\end{array}$ \\
\hline Interworking with MIPv6 & $\begin{array}{l}\text { Using MIP mobility concepts over non-standardized } \\
\text { MWiMAX upper-layer framework may lead to chal- } \\
\text { lenges related with maintaining fast handovers, long } \\
\text { signalling and handover delays and failed data con- } \\
\text { nectivity. }\end{array}$ & $\begin{array}{l}\text { MIPv6-based fast and advanced handover schemes } \\
\text { over MWiMAX are proposed in the forms of } \\
\text { FMIPv6 [49], HMIPv6 [50] and PMIPv6 [51]. }\end{array}$ \\
\hline
\end{tabular}

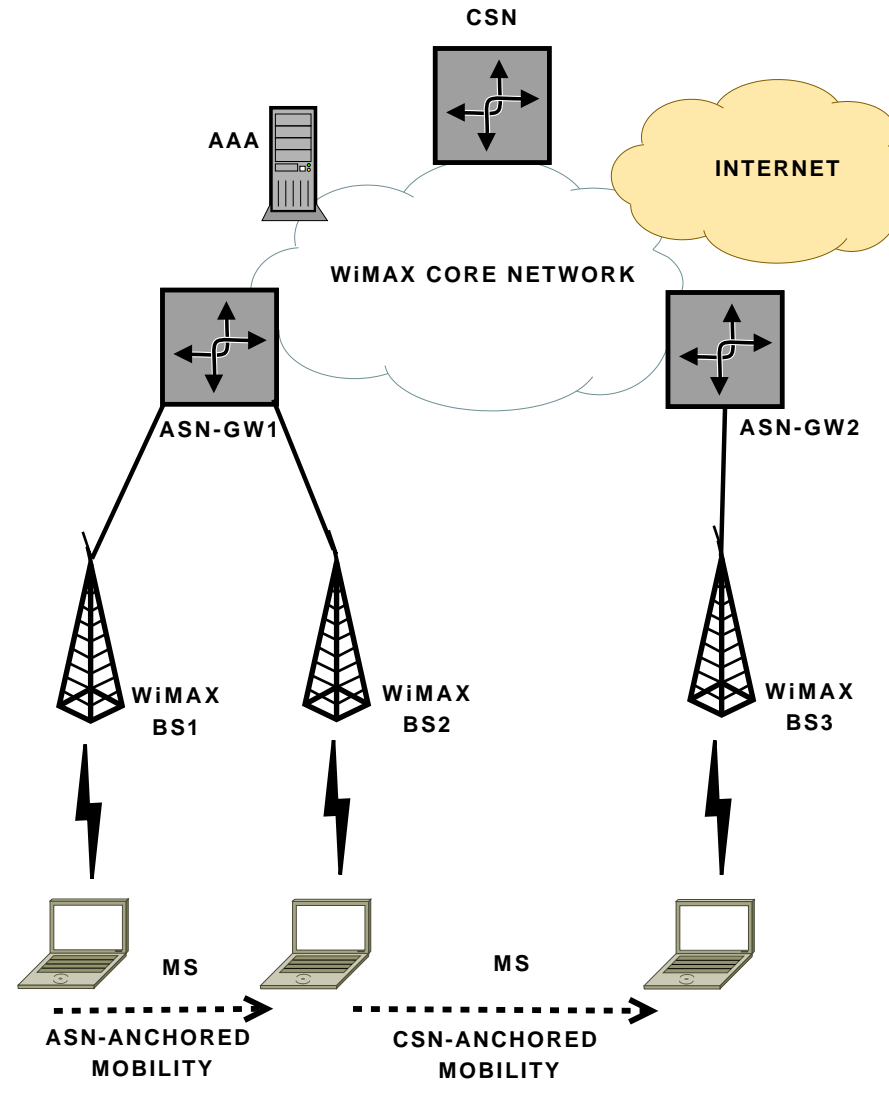

Fig. 7. ASN and CSN-Anchored Mobility in MWiMAX

handover scenario is larger, as it comprises of the delays incurred in the discovery of the new point of attachment, the establishment of the new CoA in the new subnet, and the MS notifying its new location to the HA and other correspondents [52]. In case of MWiMAX, which promises to provide nondisruptive QoS even for delay-sensitive high-speed streaming multimedia applications, large L3 handover latencies may lead to unwanted communication disruptions.

Potential Research Solutions: In view of the above-discussed ideas, reduction of the MWiMAX L3 handover latency is currently gaining attention. As, in an IP-layer handover, the BSs involved always reside in different IP-subnets, and the terminals need to perform such L2 handovers along with new IP configurations in order to maintain connectivity [53]. Thus, an L3 handover is always preceded by a well-established L2 connection. Issues on how indication of an ongoing L2 handover process could help an early L3 handover initiation by the MS are discussed in [47]. Such an approach reduces the L3 handover latency as the MS does not need to wait for the Mobile IP (MIP) router advertisement procedure, which takes a longer time. However, in case of MWiMAX environments, this scheme needs to pay further attention to such practical issues as proposing acceptable L2 triggering methodologies indicating a probable or an ongoing link layer handover activity, along with suitable timings for the L2 triggering. In MWiMAX, L2 handover triggers can originate at the MS or at the BS or even at the backbone network. In a scheme proposed in [48], anticipating a potential L2 handover activity, the SBS sends a pre-handover notification message to the corresponding access router. This helps the network layer to initiate an early L3 handover procedure, thereby reducing the handover latency. However, selecting the type of L2 triggers, whether predictive or event-based, is still an open issue. Predictive triggers, though give an early indication of a probable change in the system state, sometimes lead to false alarms as discussed in [54], and can be hazardous for L3. Event-based triggering is devoid of such problems but the advantage of early trigger initiation is absent in such cases. So, deciding upon the ideal choice and timings of L2 triggers in MWiMAX networks in order to reduce $\mathrm{L} 3$ handover latency is an open problem.

e) MAC State Migration Problem: MWiMAX HHO does not typically support MAC state transmission from the source to the destination networks. Therefore, all MAC PDUs at the source network that remain non-transmitted during the handover are discarded and new PDUs are constructed at the target network from the received IP packets after the handover is completed. However, there is always a high probability that some of the untransmitted MAC PDUs may not be recoverable [12], and resetting the MAC state in the target network can only be done by retransmissions, with the help of the higher layers, like transport or application. However, this will cause serious delays unwanted for real-time delay-sensitive applications. 
Potential Research Solutions: In order, to counter this problem, it may be possible that the serving network buffers all the IP packets [12] transmitted to the MS, such that in case of lost PDUs, the corresponding IP packets from the buffer can be aptly tunnelled to the target network over the backbone. The target MAC can accordingly reset the MAC PDUs from those. Though the buffer size required in this case is large, the handover delay would be much less.

f) Interworking with MIPv6: In MWiMAX, a major issue is supporting efficient IP mobility, particularly in case of inter-subnet movement of MSs. In order to provide unhampered and reliable QoS the IP connections should be continuously and ably maintained across the changing routers. MIPv6 supports such global IP mobility in an efficient and scalable way. In a MIP-supported mobility environment, an MS can maintain its home address throughout its movement. When under a foreign router, the MS registers a new configured care-of-address (CoA) with a home network router, which thus acts as the MS's Home Agent (HA). The HA tunnels all packets for the MS to its current location, based on its home address and the CoA. Figure 8 shows a potential Mobile IP Architecture in a MWiMAX environment. However, the large latencies occurring in MWIMAX handover cannot be reduced by MIPv6 alone, because MIPv6 mostly serves as a location and path-management protocol [53] rather than a handover management protocol. It suffers from drawbacks like long handover latencies in case of new CoA configuration and MS's location registration with the HA. Also, duplicated address detection (DAD) and long tunneling delays resulting from tunneling all packets for an MS through its HA are the major issues here [55].

Potential Research Solutions: To counter all such MIP related drawbacks, the IPv6 Forum has collaborated with the MWiMAX NWG for discussing the MIP-related problems in MWiMAX mobility and handover scenario with the goal of promoting smooth MIP connectivity over MWiMAX. Apart from the basic MIP related mobility problems discussed above, the collaboration has formulated other challenges [56][57] related to IPv4 or IPv6 adoption over the MWiMAX networks. During an inter-subnet handover, a MWiMAXenabled MS, immediately after entering a foreign network, fails to maintain further data connectivity. This is because an IP connectivity in MIPv6 is re-established only after the completion of the handover. The MS thus lacks any broadcasting or any other communication facilities for IPv6 packet exchanges, which could have facilitated the detection of appropriate routers or other nodes in the foreign network. Another serious IPv6-related problem in MWiMAX networks is the application of fast handovers over Mobile IPv6 links in such networks. As identified in [49], such fast handover techniques enables an MS to quickly detect its movement to a new subnet link and thus the MS can immediately start packet exchanges from that new link. Such handovers therefore significantly reduce the overall L3 handover latency. However, to effectively carry out such HO techniques,

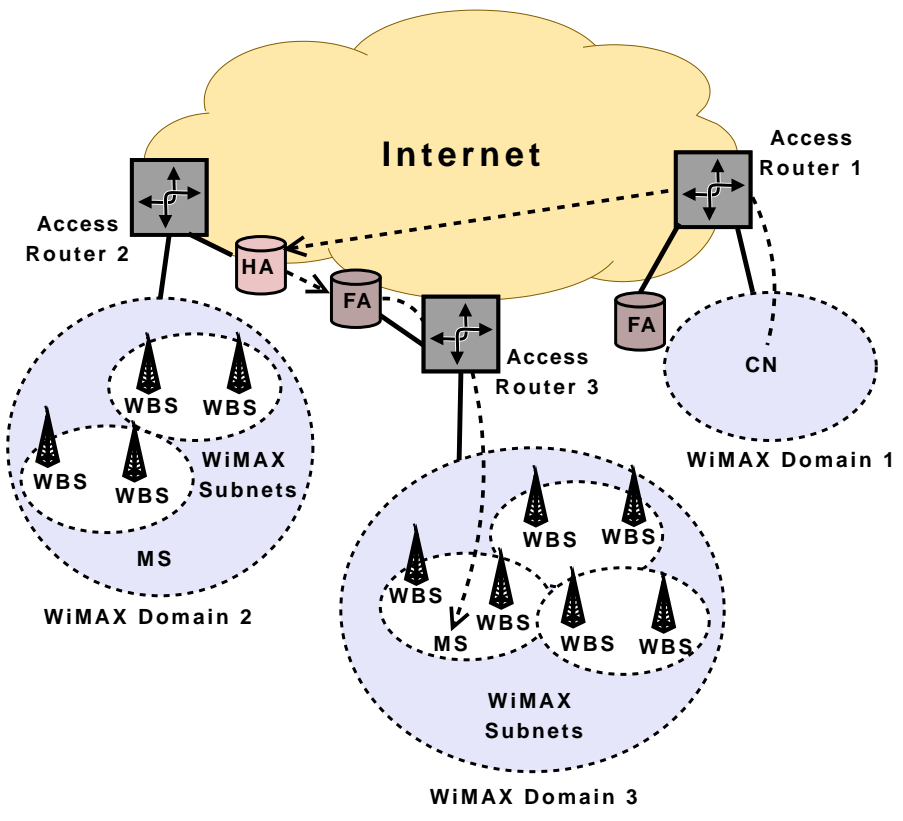

Fig. 8. Mobile IP Architecture in a MWiMAX Scenario

role of L2 triggers is important, which MIPv6 truly lacks. As pointed out in [56], MWiMAX MSs do not have the facility for multicasting of IPv6 packets after performing network re-entry during a handover activity. Owing to this, immediately after entering a new network, a MWiMAX MS has no capability whatsoever for data connectivity and suffers from drawbacks like address resolution, router discovery and DAD [56]. These problems are more relevant in MWiMAX centralized deployment architectures where the BSs may not have any MIP functionalities loaded in them. Hence, the underlying architecture between the MWiMAX BSs and routers will control the MIP adoption methodologies for MWiMAX [58]. In this context, mechanisms like FMIPv6, HMIPv6 and PMIPv6, which are also gaining importance in context to alike L3 issues in WLAN environments, are discussed to deal with the MIPv6-related problems in MWiMAX handovers.

g) Fast Handover for Mobile IPv6 (FMIPv6) in MWiMAX Mobility Scenario: FMIPv6 [49] takes care of the latency factors in MIPv6 arising out of address configuration and movement detection procedures in MIPv6. It provides a seamless HO solution based on the IPv6 address space and efficient use of L2 triggers. L2 triggers enable an FMIPv6-enabled MS to quickly detect its movement to a new subnet. FMIPv6 helps the MS to achieve its CoA even before the initiation of handover. It occurs in two possible scenarios: the predictive and reactive modes, respectively, depending on whether the L3-HO occurs after setting-up a tunnel between old and new ARs or not. A detailed explanation of the procedure is given in [49]. However, a considerable performance degrading connection disruption interval still exists between the MS being disconnected from the old AR and reconnected to the new AR. Also, in case of a MWiMAX $\mathrm{HO}$, if FMIPv6 is occurring in a reactive mode, it leads to 
increased latency and packet losses owing to absence of the tunnel.

Potential Research Solutions: In order to reduce the drawbacks and to efficiently support FMIPv6 over the MWiMAX technology, a fast FMIPv6 scheme has been proposed in [59] to facilitate MWiMAX inter-subnet handovers. Capable of operating in both predictive and reactive modes, this method uses four L2/L3 handover triggers [59] to reduce the L3 handover latencies. Each of these triggers is introduced during individual phases of access router discovery, handover preparation, handover execution and handover completion, respectively. However, the scheme lacks an effective blending between the L2 and L3 handover management messages and thus results in limited improvement of the overall performance. A new L3-HO trigger message, HO_FASTHI, transmitted by the selected TBSs to the ARs, is proposed in [60]. It contains information about MS's CoA and the previous AR. Using these information, prior to the probable HO activity, the ARs of the TBSs establishes HO tunnel with the previous AR to carry out the entire activity in a predictive mode. This enhances the overall HO performance. However, effectively setting-up the $\mathrm{HO}$ tunnels to make the $\mathrm{HO}$ run in a predictive fashion and using new L2-triggers during the FMIPv6MWiMAX HO activity are some of the issues requiring further research.

h) Hierarchical MIPv6 (HMIPv6) in MWiMAX Mobility Scenario: Alike FMIPv6, HMIPv6 is also an improved solution for MIPv6 operating in both micro and macromobility modes. It reduces the amount of signaling overhead between the MS, its correspondent node (CN) and the HA. HMIPv6 supports a special network entity called the Mobility Anchor Point (MAP), which is basically a router or a group of routers. During mobility, MAP acts as an HA to the MS and channels all traffic to the MS through the CoA. Thus, within a domain, a binding always remains between the MAP and the MSs. Detailed description of the scheme can be found in [50].

Potential Research Solutions: In the HMIPv6 handover procedure in MWiMAX, an L3 handover is initiated only after the completion of the L2 handover. The overall performance could have been much better in terms of latency and packet loss, had the L3 and L2 processes occur in parallel, utilizing timely L2 handover indications as discussed in [61]. However, as the HMIPv6 is only a localized or an intra-domain solution, scope of research is there on issues related to effectively handling MWiMAX MSs when moving outside the domain. Efficient interworking between HMIPv6 and MIPv6 in a MWiMAX scenario requires more research. Another advancement of the HMIPv6 protocol is proposed in [62]. This fast handover mechanism based on HMIPv6 over MWiMAX is termed as Partner-Assisted HMIPv6. Here with the help of another static subscriber station, called the partner station (PS), an MS could detect the presence of a neighbour BS early enough, when it feels the necessity of a handover. It might happen that an MS requiring a handover is not within the coverage area of any neighbour BSs. At this point, with the help of a PS, within the coverage area of a neighbouring BS, the MS could detect the existence of the BS early enough to initiate a handover activity. It is assumed that every PS in this context has relaying abilities and acts as a relay agent between an MS and the neighbouring BSs. The PS helps an MS to perform L3 handover early enough before the MS actually reaches the TBS. This scheme gives better results than HMIPv6 in the MWiMAX scenario in terms of latency and packet loss. However, finding suitable PSs in the neighbouring MAP domains and within the neighbouring subnet to perform pre-handover operations is an uphill task in this scheme.

i) Proxy MIPv6 (PMIPv6) in MWIMAX Mobility Scenario: A very recent proposal based on MIPv6 is the PMIPv6 mechanism, which is a network-based mobility scheme [51]. PMIPv6 provides network-based mobility management support to MSs within a localized domain and is recently getting prevalent in the WLAN environments as well. PMIPv6 introduces a new functional entity, the Proxy Mobile Agent (PMA), a kind of MIPv4 foreign agent located on the AR. The PMA acts as a relay node between the HA and the MS. The MS does not participate in any sort of mobility related signaling activities, as they are performed by the PMA instead, on behalf of the MS. A detailed discussion about PMIPv6 can be found in [51].

Potential Research Solutions: The NWG of the MWiMAX Forum has identified PMIPv6 as a mechanism aligned with the architectural direction of MWiMAX and thus as a potential solution to the MWiMAX MIPv6-related problems. An advantage of PMIPv6 in this context is, it can also be useful in scenarios where the MWiMAX operators might have interests in host-based MIPv6 solutions, in order to maintain some hosts in a network-based manner. Hence, a common infrastructure can be maintained both for the host-based and network-based mobility. Other benefits in terms of optimized HO performance offered by PMIPv6 in the MWiMAX mobility scenario are moderate HO latency, enhanced location privacy and low HO-related signaling overheads [63]. However, PMIPv6-based mobility is preferred in cases where the mobility is restricted within a domain. Room for further research exists with the application of PMIPv6 over MWiMAX network, specially combining PMIPv6 with FMIPv6 [63] and HMIPv6 to further reduce the handover latency with the help of link layer triggers.

In this context, Table VI provides a brief comparison of MIPv6 and its different advancements with respect to the handover techniques. Detailed discussion on these can be found in [64],[65] and [66]. Despite of a few attempts, further research on designing improved L3 handover frameworks is needed. Special consideration should be given to issues like effective signaling management, IP stack implementations across the MWiMAX-enabled BSs and MSs, and standardization of the design of MWiMAX convergence sub-layers facilitating fast and lossless transportation of IP packets. Also MIP-based handovers for delay-sensitive real-time traffic 
TABLE VI

BRIEF COMPARISON OF L3 HO SCHEMES

\begin{tabular}{|l||l||l||l||l|}
\hline Parameters & MIPv6 & HMIPv6 & FMIPv6 & PMIPv6 \\
\hline Complexity & Medium & High & High & Medium \\
\hline Latency & High & High & Low & Medium \\
\hline Scalability & Medium & Medium & Medium & Low \\
\hline Packets Loss & High & Medium & Medium & Medium \\
\hline Mobility & $\begin{array}{l}\text { Host- } \\
\text { based }\end{array}$ & $\begin{array}{l}\text { Host- } \\
\text { based }\end{array}$ & $\begin{array}{l}\text { Host- } \\
\text { based }\end{array}$ & $\begin{array}{l}\text { Network- } \\
\text { based }\end{array}$ \\
\hline $\begin{array}{l}\text { Signalling Over- } \\
\text { heads }\end{array}$ & High & Medium & High & Medium \\
\hline
\end{tabular}

in MWiMAX need special consideration. Ongoing MIPv6related research activities are expected to reduce the MIP handover related drawbacks and, hopefully, would be effective for both MWiMAX horizontal inter-subnet handovers as well as vertical handovers.

\section{MWIMAX CROSS-LAYER $(L 2+$ L3) HANDOVER ISSUES}

Research contributions to the MWiMAX handover framework until date have been mostly focussed on the link layer aspects. Of late, the MWiMAX NWG, along with the IPv6 forum and IETF, have initiated work on the network and upper layer implementation facets, to propose a universally accepted MWiMAX macro-mobility and handover framework. However, it is difficult to use these single-layer-based solutions to provide a promising mobility and handover support framework. The performance of such a framework will depend on the integrated performance of the individual layers, specifically the link and the network layers. Hence, optimization of MWiMAX seamless handover performance will largely depend on how effectively the link-layer (L2) and the network layer (L3) HO methodologies can be integrated without causing significant breaks in the IP connectivity between the two handovers. In comparison to the L3 challenges, significant research has been reported on such MWiMAX cross-layer issues, as explicit and imprecise lower layer HO triggers to the upper layer, see table VII. However, nothing concrete has been accepted yet. On the other hand, equally important issues like seamless integration of L2 and L3 handover management messages and two-way handover information flows, have not been much explored yet. The following sub-categories, present different potential cross-layer handover research issues in Mobile MWiMAX.

j) Explicit HO Notifications to Upper Layers: MWiMAX mobility and HO-related research activities should concentrate on proposing $\mathrm{HO}$ generic dynamic event services [53], which are triggered in time from the PHY or MAC layers and reported to the upper layers. This would result in better HO performance resulting from reduced delays and resource wastages, in comparison to situations where HO decisions are solely based on the L3 indications. For example, a fast handover process in a MIPv6 environment, improving the performance of the overall handover procedure, is discussed in [49]. However, in this case, the IP layer handover procedure gets initiated only after the completion of the L2-handover process. Thus, it increases the total handover delay, which is the sum of handover delays in both the layers.

Potential Research Solutions: L2 HO event services [69] indicate a probable L2-event marking an upcoming change in the L2-point of attachment of an MS within a particular subnet. In case of 802.16e, such indications may either be solicited or unsolicited MAC messages directly from the MAC layer, or they might be derived from other MAC management messages. Effective usage of such L2 triggers are proposed in [59], based on fast seamless interdomain handover mechanisms in IEEE 802.16 e by timely exploiting the L2 handover indicators. Multiple L2 triggers like New_Link_Detected (reports detection of a new link), Link_Handover_Impend (a L2 handover is to occur soon) and Link_Up (Link layer handover completed) are introduced in the different stages of the overall handover procedure. However, the scheme lacks an effective blending between the L2 and L3 handover management messages and thus results in limited improvement of the overall performance. So, along with generation of effective L2 event triggers, what is really needed is a meaningful correlated overlay of the IEEE 802.16e L2 and L3 layers. This would also enable L3 to effectively and successfully derive any inexplicit L2 handover indication on the fly.

k) Imprecise L2 Triggers: Timely generation of an effective L2-handover trigger is a big challenge. In regard to a MWiMAX L3-handover scenario, if a L2-trigger is not generated well in advance, then it would not be possible to achieve the expected boost in the overall performance. Ideally, an L2-handover trigger should be generated much before an onset L2-handover event, so that there remains sufficient time for the layer 3 to predict a probable handover activity and act accordingly. The effect of concurrent processing of the L2 and L3 handover mechanisms, which is an important technique to maintain a stable QoS for delay-sensitive applications, would be large, provided the L2 notifications are communicated on time.

Potential Research Solutions: Timely initiation of a L2handover trigger is well recognized as a difficult problem and hence has drawn considerable attention. As discussed in [70], an untimely generation of L2-handover trigger changes the FMIPv6 handover mode from a predictive one to a reactive one, causing a significant degradation in the entire handover performance. However, there is much scope for further research on this issue, particularly as the standardization of the MWiMAX layer-3 architecture is still an open issue. The L2 handover trigger in the form of predicted signal strength (RSSI) values, tracked periodically by an MS, has been introduced in [54]. Timely generation of such triggers always initiates the MWiMAX L3-handover activity in a predictive manner well in advance, thus minimizing packet losses. However, this scheme also suffers from unwanted MIP signaling overheads owing to false L2 handover alarms. Generation of false or untimely L2-handover alarms can 
TABLE VII

Summary of the Probable Cross-LAYer Handover IsSUES IN MWiMAX

\begin{tabular}{|l||l||l|}
\hline Issues & Effects & Proposed Research Directions \\
\hline $\begin{array}{l}\text { Explicit HO Notifications } \\
\text { to Upper Layers }\end{array}$ & $\begin{array}{l}\text { Lack of HO generic suitable dynamic event triggers } \\
\text { from MWiMAX PHY/MAC layers to the IP-laye } \\
\text { degrades HO performance as in that case the L3 HO } \\
\text { gets initiated after the completion of the L2 HO. }\end{array}$ & $\begin{array}{l}\text { Explicit L2 to L3 event triggers during the various } \\
\text { stages of the overall MWiMAX HO activity are } \\
\text { proposed in [59] for enhancing the performance. }\end{array}$ \\
\hline Imprecise L2 Triggers & $\begin{array}{l}\text { Untimely generation of L2-triggers hampers the } \\
\text { maximum boost in the HO performance. In addition, } \\
\text { false L2-triggers degrade performance. }\end{array}$ & $\begin{array}{l}\text { MSs can send the L2-HO trigger early enough to the } \\
\text { upper layers in the form of predicted RSSI values } \\
\text { [54]. }\end{array}$ \\
\hline $\begin{array}{l}\text { Seamless Integration of } \\
\text { agement Messages }\end{array}$ & $\begin{array}{l}\text { Merely overlaying the MWiMAX L2 and L2 HO } \\
\text { procedures without any effective correlation between } \\
\text { them increases the overall latency. }\end{array}$ & $\begin{array}{l}\text { Removal of related HO management messages from } \\
\text { both the MWiMAX L2 and L3 HO procedures } \\
\text { and coincidental processing of both the procedures } \\
\text { enhances the overall performance [67]-[68]. }\end{array}$ \\
\hline $\begin{array}{l}\text { Two-Way Cross-Layer } \\
\text { Handover Information } \\
\text { Flow }\end{array}$ & $\begin{array}{l}\text { Dynamic collaboration of the HO procedures of dif- } \\
\text { ferent layers with diverse functionalities is a difficult } \\
\text { task. }\end{array}$ & $\begin{array}{l}\text { Multiple event and command services to improve the } \\
\text { FMIPv6 HO support over the MWIMAX MAC [53]. }\end{array}$ \\
\hline
\end{tabular}

be a big problem in case of Mobile MWiMAX networks with moderate cell sizes. In case of high-speed mobility, it maximizes the chance of ping-pong activities. Hence, in order to achieve maximum gain in performance, triggers should be genuine and generated only when the MS or the BS becomes sure of a probable/ongoing handover activity. However, finding the ideal L2 notification time, which would maximize the gain, is itself tricky and specific to the IP-layer technologies used.

l) Seamless Integration of L2 and L3 Mobility Management Messages: Efficiently integrating the MWiMAX L2 and L3 MAC management messages poses significant challenge, particularly owing to the non-standardized IP-layer of MWiMAX architecture. Recently, a couple of hostbased IP-layer localised mobility management techniques, like FMIPv6 [49] and HMIPv6 [50], have been proposed and have drawn significant research attention from the MWiMAX community, focussing on tentative seamless merging techniques between the IP-layer protocols and the MWiMAX MAC layer mobility techniques.

Potential Research Solutions: Seamless integration of MWiMAX L2 and L3 mobility management messages requires an effective correlation between the messages of both the layers, rather than simply overlaying the L2 and L3 handover procedures as can be seen in [59]. Mere overlaying of the layers may hamper the improvement of the handover performance, as it would cause enhanced delays in processing more handover control messages. On the other hand, effectively correlating the mobility management messages of the MAC and the IP-layer can reduce the number of related messages in both the layers, and improves the overall handover delay. The schemes proposed in [67]-[68] discuss effective integration scenarios between FMIPv6 in the IP-layer and 802.16e MAC-layer mobility management techniques. Both the schemes propose integrated cross-layer design approaches, based on seamless combination of the L2 and L3 handover management messages. Removal of related management messages from both layers and coincidental processing of both the layers, have led to an improvement of the overall handover delay and reduction of resource wastages. Despite of all such research advancements, designing an universally accepted IP and MAC-layer integrated MWiMAX handover framework is still a long way to go because of such unresolved issues like choosing the best management protocol for the MWiMAX IP-layer in terms of scalability, complexity and implementation cost, effectively identifying and removing the related cross-layer mobility control messages, and keeping the QoS unhampered in a cross-layer scenario. The trade-off between improved latency, higher complexity and cost should also be taken into account.

m) Two-Way Cross-Layer Handover Information Flow: The usefulness of the two-way (back and forth) cross-layer information flow model during a handover activity has been identified in [71]. To get the maximum improvement in the overall handover performance along with the lower layer triggers (event services), explicit notifications of events from the upper layers to the lower layers (command services) are also required [72]-[73]. In comparison to the different MWiMAX cross-layering handover approaches discussed before, which use a single-way signaling technique particularly from the MAC-layer to the IP-layer, a two-way signaling scheme not only helps to achieve fast handover but also is useful enough in terms of resource utilisation. However, designing such a two-way signaling scheme is complicated because it requires the two different layers, with different functionalities and performing different tasks, to collaborate dynamically, which is undoubtedly an uphill challenge.

Potential Research Solutions: A cross-layering design approach for improving the FMIPv6 handover support over the 802.16e MAC layer technology has been proposed in [53]. Using the back and forth signaling flow model, this scheme introduces three different triggers from the L2 to L3, namely, NEW_CANDIDATE_BS_FOUND, LINK_GOING_DOWN and LINK_UP, along with LINK_SWITCH, a hint from L3 to $\mathrm{L} 2$, at the different stages of the handover activity. It is shown 
that this approach results in fast handover activity both in the predictive and reactive modes of handover. Another similar kind of approach employing two-way information flow model providing solutions for both intra-MAP (micro mobility) and inter-MAP (macro mobility) handovers in HMIPv6-based systems is proposed in [74]. A detailed description of the different event and command services, as outcomes of recent MWIMAX cross-layer handover research activities, is given in Table VIII. However, a limited attention has been focussed on a two-way notification approach and there is significant scope for research scenario in this area. Specifically, emphasis should be given on designing explicit approaches (command services) by which the upper layers (IP layer in this case) can timely notify (or hint) the MWiMAX lower layers (MAC and $\mathrm{PHY}$ ) about the processing of the application data based on MAC layer control messages. However, this could have an enhanced effect on the handover performance only if the layers are interleaved seamlessly.

As evident from the above discussion, that it is still a long way to go before an universally accepted performanceoptimized MWiMAX CLHM framework could be formulated. It should noticeably minimize the handover overheads like delays, connection drops and packet losses, both in case of MWiMAX intra and inter-technology handover scenario. Along with HMIPv6 and FMIPv6, PMIPv6 also requires more attention as a potential IP-layer technology in the MWIMAX cross-layer handover domain. On the other front, reasons like non-standardization of MWiMAX upper layers and the MWiMAX NWG not being sure about the potential architectural deployment scenario (hierarchical, flat or hybrid), bar the devising of an ideal MWiMAX CLHM as well.

\section{CONCLUSION}

Efficient support of seamless handover management activity is an important requirement for communication technologies that are intended to be universally accepted in next-generation communication systems. Although MWiMAX has a number of attractive features, its handover framework is not free from drawbacks and has attracted significant research attention. This paper has not only identified the diversified MAC layer and potential network layer handover issues in MWiMAX, but also has highlighted those cross-layer $(\mathrm{L} 2+\mathrm{L} 3)$ challenges that demand more attention. Out of these, the MAC-layer HHO issues related to the reduction and optimization of scanning activities and inter-handover CDT are still considered to be wide open, as the MWiMAX Forum has not reached a definite conclusion regarding whether and how to modify the existing standard to incorporate the changes suggested to date. On the other hand, the issues identified in Table III, on optimization of the network re-entry activities, load distributions, and those on the MDHO and FBSS presented in Table IV, have not yet attracted much research attention. Moving up the ladder, crosslayer challenges have gained more attention than the solely layer 3 ones. This could be because the overall macro-mobility handover performance depends jointly on the performance of the L2 and L3 handovers rather than only on the L3 handover. However, even then, cross-layer issues like seamless integration of L2 and L3 handover management messages and efficient bidirectional flow of these messages, have been less visited than the other issues and need more attention in order to devise a good MWiMAX CLHM framework. A standardization of the MWiMAX L3 framework would help to achieve this. Attention should be given to choosing the best option for MIPv6 (e.g. FMIPv6, HMIPv6 or PMIPv6), to provide seamless handover performance for high-speed real-time multimedia applications. Although, it appears that PMIPv6 is the most promising option, being the mechanism closely aligned with the architectural direction of MWiMAX, since nothing has been decided yet, this is still a wide open area for investigation.

APPENDIX A

List OF ACRONYMS USED IN THE PAPER

\begin{tabular}{|l||l|}
\hline Acronyms & Full Form \\
\hline ABS & Anchor Base Station \\
\hline AHOP & Actual Handover Phase \\
\hline AR & Access Router \\
\hline AS & Active Set \\
\hline ASN & Access Service Network \\
\hline ASN_GW & Access Network Gateway \\
\hline BS & Base Station \\
\hline BSID & Base Station Identification \\
\hline B3G & Beyond 3G \\
\hline CDMA & Code Division Multiple Access \\
\hline CDT & Connection Disruption Time \\
\hline CID & Connection Identification \\
\hline CINR & $\begin{array}{l}\text { Carrier to Interference + Noise Ra- } \\
\text { tio }\end{array}$ \\
\hline CLHM & $\begin{array}{l}\text { Cross-Layer Handover Manage- } \\
\text { ment }\end{array}$ \\
\hline CoA & Care-of-Address \\
\hline CN & Correspondent Node \\
\hline CS & Candidate Set \\
\hline CSN & Connectivity Services Network \\
\hline DAD & Duplicate Address Detection \\
\hline DCD & Downlink Channel Descriptor \\
\hline DL & Downlink \\
\hline DL_MAP_IE & $\begin{array}{l}\text { Downlink MAP Information Ele- } \\
\text { ment }\end{array}$ \\
\hline DS & Diversity Set \\
\hline eNB & $\begin{array}{l}\text { E-UTRAN (UMTS Terrestrial Ra- } \\
\text { dio Access Networks) Node B }\end{array}$ \\
\hline EV-DO & Evolution-Data Optimized \\
\hline FA & Frequency Assignments \\
\hline FBSS & Fast Base Station Switching \\
\hline FMIPv6 & Fast Handovers for MIPv6 \\
\hline FUSC & \begin{tabular}{l} 
Fully Used Sub-Channelization \\
\hline
\end{tabular} \\
\hline
\end{tabular}


TABLE VIII

Event And COMmand Triggers FOR MWiMAX CROSS-LAYER HO

\begin{tabular}{|c|c|c|c|c|c|}
\hline $\begin{array}{l}\text { Type of } \\
\text { Services }\end{array}$ & Name of Services / Proposed w.r.t & & From & To & Description of Services \\
\hline Event & $\begin{array}{l}\text { NEW_LINK_DETECTED } \\
\text { NEW_CANDIDATE_BS_FOUND. } \\
\text { Proposed w.r.t: FMIPv6 }\end{array}$ & I & L2 & L3 & $\begin{array}{l}\text { Reports the L3 about the detection of a new BS for a } \\
\text { potential HO activity. } \\
\text { Purpose: To learn about the Access Router (AR) associated } \\
\text { with the newly detected BS. }\end{array}$ \\
\hline Event & $\begin{array}{l}\text { LINK_HANDOVER_IMPEND } \\
\text { LINK_GOING_DOWN. } \\
\text { Proposed w.r.t: FMIPv6 }\end{array}$ & I & L2 & L3 & $\begin{array}{l}\text { Reports the L3 about imminent execution of an } \mathrm{HO} \text { activity. } \\
\text { Purpose: To indicate the L } 3 \text { to get prepared for a likely } \mathrm{HO} \\
\text { procedure. }\end{array}$ \\
\hline Event & $\begin{array}{l}\text { LINK_UP. } \\
\text { Proposed w.r.t: FMIPv6 }\end{array}$ & & L2 & L3 & $\begin{array}{l}\text { Reports the L3 that the link-layer connection establishment } \\
\text { with the new BS is accomplished successfully. } \\
\text { Purpose: Enables the L3 to check whether it has really } \\
\text { moved to the predicted target network. }\end{array}$ \\
\hline Event & $\begin{array}{l}\text { RSSI / SNR values. } \\
\text { Not incorporated yet. }\end{array}$ & & L2 & L3 & $\begin{array}{l}\text { A low value reports the L3 that a } \mathrm{HO} \text { is expected within a } \\
\text { certain } \Delta \mathrm{t} \text { time. } \\
\text { Purpose: Enables the L3 to start the L3-HO procedure } \\
\text { early enough even before the L2-HO. }\end{array}$ \\
\hline Event & $\begin{array}{l}\text { HO-NOTIF. } \\
\text { Proposed w.r.t: FMIPv6. } \\
\text { L3_HO-INITIATE. } \\
\text { Proposed w.r.t: HMIPv6. }\end{array}$ & & L2 & L3 & $\begin{array}{l}\text { Reports the L3 of an impending HO activity and contains } \\
\text { the information of the SBS recommended TBSs and MAC } \\
\text { address of MS. } \\
\text { Purpose: Enables the initiation of the L3-HO. }\end{array}$ \\
\hline Event & $\begin{array}{l}\text { L3_Buffer-INITIATE. } \\
\text { Proposed w.r.t: HMIPv6 }\end{array}$ & & L2 & L3 & $\begin{array}{l}\text { During an intra-domain HO, MAP reports the MS's LCoA } \\
\text { to the new AR after receiving the local binding update from } \\
\text { the old AR. } \\
\text { Purpose: Enables the new AR to initiate buffering for } \\
\text { the MS during the impending HO phase. }\end{array}$ \\
\hline Event & $\begin{array}{l}\text { L2_HO-COMPLETE. } \\
\text { Proposed w.r.t: HMIPv6 }\end{array}$ & & L2 & L3 & $\begin{array}{l}\text { TBS reports the completion of the L2-HO activity to the } \\
\text { new AR. } \\
\text { Purpose: Initiates the completion of an L3 HO and } \\
\text { channelization of all buffered packets for the MS from the } \\
\text { new AR. }\end{array}$ \\
\hline Event & $\begin{array}{l}\text { LINK_LOST. } \\
\text { Not incorporated yet. }\end{array}$ & & L2 & L3 & $\begin{array}{l}\text { Reports the TBS of a ping-pong effect. } \\
\text { Purpose: Initiates the AR associated with the new TBS to } \\
\text { flush back all the buffered data to the old SBS. }\end{array}$ \\
\hline Event & $\begin{array}{l}\text { HO-FASTHI. } \\
\text { Proposed w.r.t: FMIPv6 }\end{array}$ & & L2 & L3 & $\begin{array}{l}\text { A fast tunnelling message, through which, the selected TBSs } \\
\text { transmit the NCoA of the MS to the associated ARs. } \\
\text { Purpose: Initiates the associated ARs to validate the } \\
\text { NCoA of the MS and sets up a tunnel with the PAR if the } \\
\text { NCoA is valid. }\end{array}$ \\
\hline Command & $\begin{array}{l}\text { LINK_SWITCH. } \\
\text { Proposed w.r.t: FMIPv6 }\end{array}$ & & L3 & $\mathrm{L} 2$ & $\begin{array}{l}\text { Forces an MS to perform an L3 switch from under the } \\
\text { current SBS to the TBS. } \\
\text { Purpose: The L3 asks the L2 to transmit the MOB_HO-IND } \\
\text { command asap. }\end{array}$ \\
\hline
\end{tabular}

\begin{tabular}{|l|l|}
\hline \multicolumn{2}{|l|}{ continued from previous page / column } \\
\hline 4G & Fourth-Generation \\
\hline HA & Home Agent \\
\hline HHO & Hard Handover \\
\hline HMIPv6 & Hierarchical MIPv6 \\
\hline HO & Handover \\
\hline HSDPA & $\begin{array}{l}\text { High-Speed Downlink Packet Ac- } \\
\text { cess }\end{array}$ \\
\hline \multicolumn{1}{|c|}{ continued on next page / column } \\
\hline
\end{tabular}

\begin{tabular}{|l||l|}
\hline \multicolumn{2}{|l|}{ continued from previous page / column } \\
\hline IEEE & $\begin{array}{l}\text { Institute of Electrical and Electron- } \\
\text { ics Engineers }\end{array}$ \\
\hline IETF & Internet Engineering Task Force \\
\hline L2 & Layer 2 \\
\hline L3 & Layer 3 \\
\hline LTE & Long Term Evolution \\
\hline \multicolumn{2}{|c|}{ continued on next page / column } \\
\hline
\end{tabular}




\begin{tabular}{|c|c|}
\hline \multicolumn{2}{|c|}{ continued from previous page / column } \\
\hline MA & Mobility Agent \\
\hline MAP & Mobility Anchor Point \\
\hline MBB & Make-Before-Break \\
\hline MDHO & Macro-Diversity Handover \\
\hline MIP & Mobile IP \\
\hline MIPv6 & Mobile IP version 6 \\
\hline MIPv4 & Mobile IP version 4 \\
\hline MME & Mobility Management Entity \\
\hline MOB_ASC-REP & Association Result Report \\
\hline MOB_HO-IND & Mobile Handover Indication \\
\hline MOB_MSHO-REQ & Mobile Station Handover Request \\
\hline MOB_BSHO-REQ & Base Station Handover Request \\
\hline MOB_BSHO-RSP & Base Station Handover Response \\
\hline MOB_NBR-ADV & Mobile Neighbour Advertisement \\
\hline MOB_SCN-REQ & $\begin{array}{l}\text { Scanning Interval Allocation Re- } \\
\text { quest }\end{array}$ \\
\hline MOB_SCN-RSP & $\begin{array}{l}\text { Scanning Interval Allocation Re- } \\
\text { sponse }\end{array}$ \\
\hline MOB_SCN-REP & Scanning Result Report \\
\hline MS & Mobile Station \\
\hline MWiMAX & $\begin{array}{l}\text { Mobile Wireless Interoperability } \\
\text { for Microwave Access (Mobile } \\
\text { WiMAX) }\end{array}$ \\
\hline NBS & Neighbouring Base Station \\
\hline NTAP & $\begin{array}{lll}\text { Network Topology } & \text { Acquisition } \\
\text { Phase } & & \\
\end{array}$ \\
\hline NWG & Network Working Group \\
\hline OFDM & $\begin{array}{ll}\text { Orthogonal } & \text { Frequency-Division } \\
\text { Multiplexing } & \\
\end{array}$ \\
\hline OFDMA & $\begin{array}{l}\text { Orthogonal Frequency-Division } \\
\text { Multiple Access }\end{array}$ \\
\hline PDU & Protocol Data Unit \\
\hline PMA & Proxy Mobile Agent \\
\hline PMIPv6 & Proxy MIPv6 \\
\hline PS & Partner Station \\
\hline PUSC & Partially Used Sub-Channelization \\
\hline QoS & Quality of Service \\
\hline RAN & Radio Access network \\
\hline RNG_REQ & Ranging Request \\
\hline RNG_RSP & Ranging Response \\
\hline RRM & Radio Resource Management \\
\hline RSSI & $\begin{array}{l}\text { Received Signal Strength Indica- } \\
\text { tion }\end{array}$ \\
\hline SAE & System Architecture Evolution \\
\hline SBS & Serving Base Station \\
\hline SCR & Spare Capacity Report \\
\hline SHO & Soft Handover \\
\hline TBS & Target Base Station \\
\hline $3 \mathrm{G}$ & Third-Generation \\
\hline $\mathrm{UCD}$ & Uplink Channel Descriptor \\
\hline$\overline{\mathrm{UL}}$ & Uplink \\
\hline
\end{tabular}

\begin{tabular}{|l||l|}
\hline \multicolumn{2}{|l|}{ continued from previous page / column } \\
\hline UMTS & $\begin{array}{l}\text { Universal Mobile Telecommunica- } \\
\text { tions System }\end{array}$ \\
\hline VoIP & Voice-Over-Internet Protocol \\
\hline WLAN & Wireless Local Area Networks \\
\hline WiMAX & $\begin{array}{l}\text { Wireless Interoperability for Mi- } \\
\text { crowave Access }\end{array}$ \\
\hline
\end{tabular}

\section{ACKNOWLEDGMENT}

The authors would like to thank Dr. Allan McInnes, Department of Electrical and Computer Engineering, University of Canterbury for stimulating discussions and helpful comments, and the reviewers for suggestions, which improved the content and style of the paper.

\section{REFERENCES}

[1] IEEE 802.16-2004: IEEE Standard for Local and Metropolitan Area Networks-Part 16: Air Interface for Fixed Broadband Wireless Access Systems.

[2] IEEE 802.16e-2005: IEEE Standard for Local and Metropolitan Area Networks-Part 16: Air Interface for Fixed and Mobile Broadband Wireless Access Systems.

[3] Ed Agis et. al. Global, Interoperable Broadband Wireless Networks: Extending WiMAX Technology to Mobility. Intel Technology Journal, 8(3):173-187, 2004.

[4] R. Q. Hu et al. On the Evolution of Handoff Management and Network Architecture in WiMAX. In Proc. of IEEE Mobile WiMAX Symposium, pages 144-149, Florida, USA, 25-29 March 2007.

[5] Requirements for Evolved UTRA (E-UTRA) and Evolved UTRAN (EUTRAN) V7.3.0. $3^{\text {rd }}$ Generation Partnership Project, March 2006 (TR 25.913). http://www.3gpp.org

[6] Z. Becvar and J. Zelenka. Handovers in Mobile WiMAX. Research in Telecommunication Technology, 1:147-150, 2006, ISBN 80-214-3243-8.

[7] Mobile WiMAX-Part I: A Technical Overview and Performance Evaluation. White Paper, WiMAX Forum, August 2006. http://www.wimaxforum.org/news/downloads/ Mobile_WiMAX_Part1_Overview_and_Performance.pdf

[8] CDMA 2000 High Rate Packet Data Air Interface Specification. 3GPP2 C.SO024-A, Standard, ver. 2.0, 27 October 2000. http://www.3gpp2.org/public_html/specs/C.SO024_v2.0.pdf

[9] Physical Layer Aspects of UTRA High Speed Downlink Packet Access. 3GPP TR 25.848, 3GPP Specification Series. http://www.3gpp.org/ftp/Specs/html-info/25848.htm

[10] Technical Specification Group Radio Access Network: Physical layerGeneral description. 3GPP TR 25.201, 3GPP Specification Series. http://www.3gpp.org/ftp/Specs/html-info/25201.htm

[11] Mobile WiMAX-Part II: A Comparative Analysis. White Paper, WiMAX Forum, May 2006. http://www.wimaxforum.org/news/downloads/ Mobile_WiMAX_Part2_Comparative_Analysis.pdf

[12] S. Das et al. System Aspects and Handover Management for IEEE 802.16e. Bell Labs Technical Journal, 11(1):123-142, 2006.

[13] B. Gage et al. WiMAX: Untethering the Internet User. Nortel Technical Journal, 2:31-38, July 2005.

[14] A. R. Gana. Wireless Communication System for Land Seismic Operations: A Feasibility Study. Master of Science Thesis, Norwegian University of Science and Technology, July 2008.

[15] Mobile Broadband Wireless Access (MBWA). IEEE 802.20 Working Group Permanent Documents, http://www.ieee802.org/20/index.html.

[16] iBurst Technical Profile. Kyocera Global Site, White Paper. http://global.kyocera.com/prdct/telecom/office/iburst/technicaloverview.pdf

[17] IEEE 802.16 Task Group m (TGm). http://wirelessman.org/tgm

[18] F. Wang et al. Mobile WiMAX Systems: Performance and Evaluation. IEEE Communications Magazine, 46(10):41-49, October 2008.

[19] D. H. Lee, K. Kyamakya and J. P. Umondi. Fast Handover Algorithm for IEEE 802.16e Broadband Wireless Access System. In Proc. of $1^{\text {st }}$ International Symposium on Wireless Pervasive Computing, Phuket, Thailand, 16-18 January 2006.

[20] Sayan K. Ray et al. Hybrid Predictive Base Station (HPBS) Selection Procedure in IEEE 802.16e-Based WMAN. In Proc. of Australasian Telecommunication Networks and Applications Conference (ATNAC), pages 93-98, Christchurch, New Zealand, 2-5 December 2007. 
[21] R. Rouil and N. Golmie. Adaptive Channel Scanning for IEEE 802.16e. In Proc. of IEEE Military Communications Conference (MILCOM), pages 1-6, Washington D.C, USA, 23-25 October 2006.

[22] S. Cho et al. Hard Handoff Scheme Exploiting Uplink and Downlink Signals in IEEE 802.16e Systems. In Proc. of IEEE Vehicular Technology Conference (VTC), vol. 3, pages 1236-1240, Melbourne, Australia, Spring 2006.

[23] S. Choi et al. Fast handover scheme for Real-Time Downlink Services in IEEE 802.16e BWA System. In Proc. of Vehicular Technology Conference (VTC), vol. 3, pages 2028-2032, Stockholm, Sweden, Spring 2005.

[24] Y. Saifullah and A. Reid. Low Latency Handover. IEEE 802.16 Broadband Wireless Access Working Group Project, IEEE C802.16g-05/18r0, 29 April 2005. http://wirelessman.org/netman/contrib/C80216g05_018r0.pdf

[25] H. Kang, C. Koo and J. Son. Resource Retain Time for Handover or Ping Pong Call Recovery. IEEE 802.16 Broadband Wireless Access Working Group Project, IEEE C802.16e-04/55r2, 17 May 2004. http://wirelessman.org/tge/contrib/C80216e-04_55r2.pdf

[26] B. Meandzija and P. Iyer. Minimizing IP Connectivity Delay during Network Re-Entry. IEEE 802.16 Broadband Wireless Access Working Group Project, IEEE C802.16e-04/151r1, 25 June 2004. http://wirelessman.org/tge/contrib/C80216e-04_151.pdf

[27] T. Casey, N. Veselinovic and R. Jantti. Base Station Controlled Load Balancing with Handovers in Mobile WiMAX. In Proc. of IEEE $19^{\text {th }}$ International Symposium on Personal, Indoor and Mobile Radio Communications (PIMRC), pages 1-5, Cannes, France, 15-18 September 2008.

[28] WiMAX Forum Network Architecture-Stage 2: Architecture Tenets, Reference Model and Reference Points-Release 1, Version 1.2. WiMAX Forum Network Working Group, WiMAX Forum, January 2008.

[29] P. Barber, Revision of Handover Mechanism for Mobility Enhancement. IEEE 802.16 Broadband Wireless Access Working Group Project, IEEE C802.16e-03/57, 30 October 2003. http://wirelessman.org/tge/contrib/C80216e-03_57.pdf

[30] H-G. Choi, J. Jeong and H. Choo. CTBS: Cost-Effective Target BS Selection Scheme in IEEE 802.16e Networks. In Proc. of Australasian Telecommunication Networks and Applications Conference (ATNAC), pages 99-103, Christchurch, New Zealand, 2-5 December 2007.

[31] H. Fattah and H. Alnuweiri. A New Handover Mechanism for IEEE 802.16e Wireless Networks. In Proc. of International Wireless Communications and Mobile Computing Conference (IWCMC), pages 661-665, Crete Island, Greece, 6-8 August 2008.

[32] N. Banerjee, K. Basu and S. K. Das. Handoff Delay Analysis in SIP-based Mobility Management in Wireless Networks. In Proc. of International Parallel and Distributed Processing Symposium (IPDPS), pages 224-231, Nice, France, 22-26 April 2003.

[33] W. Jiao, P. Jiang and Y. Ma. Fast Handover Scheme for Real-Time Applications in Mobile WiMAX. In Proc. of IEEE International Conference on Communications (ICC), pages 6038-6042, Glasgow, Scotland, 24-28 June 2007.

[34] S. K. Ray, K. Pawlikowski and H. Sirisena, A Fast MAC-Layer Handover for an IEEE 802.16e-Based WMAN. In Proc. of $3^{\text {rd }}$ International Conference on Access Networks (Accessnets), Las Vegas, USA, 15-17 October 2008.

[35] H-A (Paul) Lin. Handoff for Multi-interfaced 802 Mobile Devices. IEEE P802 Handoff ECSG, Document, May 2003. www.ieee802.org/21/archived_docs/Documents/Submissions/ Handoff_for_Multi_interfaced_MN.pdf

[36] H. Kang et al. Ping Pong Call Resuming Procedure during HO. IEEE 802.16 Broadband Wireless Access Working Group Project, IEEE 802.16e/03-26rl, 11 March 2004. http://www.ieee802.org/16/tge/contrib/C80216e-04_26r1.pdf

[37] H. Velayos, V. Aleo and G. Karlsson. Load Balancing in Overlapping Wireless LAN Cells. In Proc. of IEEE International Conference on Communications (ICC), vol. 7, pages 3833-3836, Paris, France, 20-24 June 2004.

[38] WiMAX Forum Network Architecture-Stage 3: Detailed Protocols and Procedures-Release 1.1.2. WiMAX Forum Network Working Group, WiMAX Forum, 11 January 2008.

[39] T. Casey. Base Station Controlled Load Balancing with Handovers in Mobile WiMAX. Master of Science Thesis, Helsinki University of Technology, 10 January 2008 .

[40] S. H. Lee and Y. Han. A Novel Inter-FA Handover Scheme for Load Balancing in IEEE 802.16e System. In Proc. of IEEE Vehicular Technology Conference (VTC), pages 763-767, Dublin, Ireland, Spring, 22-25 April 2007.
[41] K. Raivio. Analysis of Soft Handover Measurements in 3G Network. In Proc. of $9^{\text {th }}$ ACM International Symposium on Modeling Analysis and Simulation of Wireless and Mobile Systems (MSWiM), pages 330-337, Terromolinos, Spain, 2-6 October 2006.

[42] O. C. Ozdural. Performance-Improving Techniques for Wireless Systems. PhD Thesis 2007, Oregon State University.

[43] A. Ulvan. Using the Relative Thresholds in Handover Procedure. IEEE 802.16 Broadband Wireless Access Working Group Project, IEEE C802.16j-07/086, 8 January 2006. http://wirelessman.org/relay/contrib/C80216j-07_086.pdf

[44] J. Pinola and K. Pentikousis. Mobile WiMAX. The Internet Protocol Journal, Cisco Systems, 11(2):19-35, June 2008.

[45] K. Etemad. Overview of Mobile WiMAX Technology and Evolution. IEEE Communications Magazine, 46(10):31-40, October 2008.

[46] P. Iyer et. al. All-IP Network Architecture for Mobile WiMAX. In Proc. of IEEE Mobile WiMAX Symposium, pages 54-59, Florida, USA, 25-29 March 2007.

[47] C-T. Chou and K.G. Shin. An Enhanced Inter-Access Point Protocol for Uniform Intra and Inter Subnet Handoffs. IEEE Transactions on Mobile Computing, 4(4):321-334, July-August 2005.

[48] Y. H. Choi, Y. U. Chung and H. Lee. Early Handover Trigger. IEEE 802.16 Broadband Wireless Access Working Group Project, IEEE C802.16j-07/150, 8 January 2007. http://wirelessman.org/relay/contrib/C80216j-07_150.pdf

[49] R. Koodli. Mobile IPv6 Fast Handovers. MIPSHOP Working Group, Internet-Draft, draft-ietf-mipshop-fmipv6-rfc4068bis-07.txt, 17 April 2008. http://tools.ietf.org/html/draft-ietf-mipshop-fmipv6-rfc4068bis-07

[50] H. Soliman et. al. Hierarchical Mobile IPv6 Mobility Management (HMIPv6). RFC 4140, 2005.

[51] S. Gundavelli et. al. Proxy Mobile IPv6. NETLMM WG, InternetDraft, draft-sgundave-mip6-proxymip6-01, 5 January 2007. http://tools.ietf.org/html/draft-sgundave-mip6-proxymip6-01

[52] N. Montavont and T. Noel. Handover Management for Mobile Nodes in IPv6 Networks. IEEE Communication Magazine, 40(8):38-43, August 2002.

[53] Y-H. Han et. al. A Cross-Layering Design for IPv6 Fast Handover Support in an IEEE 802.16e Wireless MAN. IEEE Network, 21(6):5462, November-December 2006.

[54] Y-H. Choi et. al. Cross-Layer Handover Optimization Using Linear Regression Model. In Proc. of International Conference on Information Networking (ICOIN), pages 1-4, Busan, Korea, 23-25 January 2008.

[55] C-K. Chang. A Mobile-IP based Mobility System for Wireless Metropolitan Area Networks. In Proc. of International Conference Workshops on Parallel Processing (ICPPW), pages 429-435, Oslo, Norway, 14-17 June 2005.

[56] J. Jee et al. 16ng Problem Statement. Network Working Group, InternetDraft, draft-jee-16ng-problem-statement-02.txt, 16 October 2005. http://bgp.potaroo.net/ietf/all-ids/draft-jee-16ng-problem-statement$02 . t x t$

[57] J. Jee et al. Mobile IPv4 Fast Handovers for 802.16e networks. Network Working Group, Internet-Draft, draft-jee-mip4-fh80216e-00.txt, 11 October 2005. http://www.potaroo.net/ietf/idref/draft-jee-mip4-fh80216e

[58] M-K. Shin, J-M. Moon and Y-H. Han. Scenarios and Considerations of IPv6 in IEEE 802.16 Networks. draft-shin-ipv6-ieee802.16-02, February 2006.

[59] H. J. Jang et. al. Mobile IPv6 Fast Handovers over IEEE 802.16e Networks. MIPSHOP Working Group, Internet-Draft, draft-ietf-mipshopfh80216e-07.txt, 10 March 2008. http://tools.ietf.org/html/draft-ietfmipshop-fh80216e-07

[60] J. Kim, J. Jeong and H. Choo. An Efficient Handover Scheme with PreConfigured Tunneling in IEEE 802.16e Systems. In Proc. of Australasian Telecommunication Networks and Applications Conference (ATNAC), pages 408-413, Christchurch, New Zealand, 2-5 Deember 2007.

[61] D-G. Kim, H-J. Shin and D-R. Shin. A Network-based Handover Scheme for Hierarchical Mobile IPv6 over IEEE 802.16e. In Proc. of $10^{\text {th }}$ International Conference on Advanced Communication Technology (ICACT), vol. 1, pages 468-472, Gangwon-Do, South Korea, 17-20 Feb. 2008.

[62] Y-S. Chen et al. A Cross-Layer Partner-Assisted Handoff Scheme for Hierarchical Mobile IPv6 in IEEE 802.16e Systems. In Proc. of IEEE Wireless Communications and Networking Conference (WCNC), pages 2669-2674, Las Vegas, USA, 31 March-03 April 2008.

[63] J. Lei and X. Fu. Evaluating the Benefits of Introducing PMIPv6 for Localized Mobility Management. In Proc. of International Wireless Communications and Mobile Computing Conference (IWCMC), pages 74-80, Crete Island, Greece, 6-8 August 2008. 
[64] X. P-Costa, M. T-Moreno and H. Hartenstein. A Performance Comparison of Mobile IPv6, Hierarchical Mobile IPv6, Fast Handovers for Mobile IPv6 and their Combination. ACM SIGMOBILE Mobile Computing and Communications Review, 7(4):5-19, October 2003.

[65] J. Lei and X. Fu. Evaluating the Benefits of Introducing PMIPv6 for Localized Mobility Management. Technical Report, University of Gottingen, Germany, June 2007.

[66] Z. Zhang et al. Performance Comparison of Mobile IPv6 and Its Extensions. In Proc. of International Conference on Wireless Communications, Networking, and Mobile Computing (WiCom), pages 1805-1808, 21-25 September 2007.

[67] Y-W. Chen and F-Y. Hsieh. A Cross Layer Design for Handoff in 802.16e Network with IPv6 Mobility. In Proc. of IEEE Wireless Communications and Networking Conference (WCNC), pages 3844-3849, Hong Kong, 11-15 March 2007.

[68] J. Park, D-H. Kwon and Y-J. Suh. An Integrated Handover Scheme for Fast Mobile IPv6 Over IEEE 802.16e Systems. In Proc. of Vehicular Technology Conference (VTC), pages 1-5, Montreal, Canada, Fall, 2528 September 2006.

[69] L-A. Larzon, U. Bodin and O. Schelen. Hints and Notifications. In Proc. of IEEE Wireless Communications and Networking Conference (WCNC), vol. 2, pages 635-641, Florida, USA, 17-21 March 2002.

[70] J. Kempf, J. Wood and G. Fu. Fast Mobile IPv6 Handover Packet Loss Performance: Measurement for Emulated Real Time Traffic. In Proc. of IEEE Wireless Communications and Networking Conference (WCNC), vol. 2, pages 1230-1235, Louisiana, USA, 16-20 March 2003.

[71] V. Srivastava and M. Motani. Cross-Layer Design: A Survey and the Road Ahead. IEEE Communication Magazine, 43(12):112-119, December 2005.

[72] F. Foukalas, V. Gazis and N. Alonistioti. Cross-Layer Design Proposals For Wireless Mobile Networks: A Survey and Taxonomy. IEEE Communications Surveys and Tutorials Journal, 10(1):70-85, 1st Quarter 2008.

[73] M. Shariat, A. U. Quddus, S. A. Ghorashi and R. Tafazolli. Schedulling as an Important Cross-Layer Operation for Emerging Broadband Wireless Systems. IEEE Communications Surveys and Tutorials Journal, 11(2):74-86, 2nd Quarter 2009.

[74] Y. Zhang, F. Liu and X. Wang. A Cross-Layer Fast Handover Mechanism for IEEE 802.16e Networks with HMIPv6 Mobility. In Proc. of Workshop on Power Electronics and Intelligent Transportation System (PEITS), pages 3-7, Guangzhou, China, 4-5 August 2008.

Sayan Kumar Ray Sayan Kumar Ray has received the Bachelor of Engineering and Master of Technology degrees in Computer Science and Engineering in 1999 and 2002, respectively, from Gulbarga University, India and University of Calcutta, India. He is currently a Ph.D student in the Department of Computer Science and Software Engineering at the University of Canterbury, New Zealand. His research interests include performance analysis, mobility and QoS management of high-speed mobile broadband wireless networks.

Krzysztof Pawlikowski Krzysztof Pawlikowski is a Professor of Computer Science at the University of Canterbury, in Christchurch, New Zealand. He received a $\mathrm{Ph} . \mathrm{D}$ degree in Computer Engineering from Gdansk University of Technology, Poland, and worked at that University until February 1983. The author of over 160 journal and conference papers, and four books, Prof. Pawlikowski has given invited lectures at over 80 universities and research institutes in Asia, Australia, Europe and North America. He was the Humboldt Research Fellow (Germany) in 1983-84 and 1999, and a Visiting Professor at universities in Austria, Australia, Italy, Germany and the USA. His research interests include performance modelling of multimedia telecommunication networks, teletraffic modelling, methodologies of discrete-event computer simulation and distributed processing.
Harsha Sirisena Harsha Sirisena has a B.Sc.(Eng) (Honors) degree from the University of Ceylon and a Ph.D. degree from the University of Cambridge. $\mathrm{He}$ is a Professor of Electrical and Computer Engineering at the University of Canterbury, and has held visiting appointments at Australian National University, Lund University, National University of Singapore, University of Minnesota and University of Western Australia. He has research interests in Next Generation Networks, including Quality of Service, Mobility Management, Resiliency and Security. He is a Senior Member of the IEEE and a Member of the IET. 